\title{
Verdampfungsgleichgewichte von Mehrstoffgemischen VI: Ternäre Systeme mit Mischungslücke
}

\author{
Von Rolf HaAse \\ Aus dem Physikalisch-chemischen Institut der Universität Marburg \\ (Z. Naturforschg. 5 a, 109-124 [1950]; eingegangen am 17. März 1949)
}

\begin{abstract}
Die Koexistenz- und Stabilitätsbedingungen für Dreistoffsysteme werden durch die Fläche der Freien Enthalpie geometrisch veranschaulicht. Dadurch gelangt man zum Begriff der Gleichgewichtskurve für zwei flüssige Schichten (Entmischungs- oder Löslichkeitskurve) und des kritischen Entmischungspunktes. Die Gleichungen für den kritischen Entmischungspunkt bei gegebenem Druck und gegebener Temperatur werden nach $\mathrm{Gib} \mathrm{b} \mathrm{s}$ abgeleitet und diskutiert. Das Zustandekommen der Gleichgewichte zweier Flüssigkeiten mit Dampf wird geometrisch erläutert. Vollständige Gleichgewichtsdiagramme für ternäre Systeme mit Mischungslücke werden an Hand von Beispielen besprochen. Allgemeine Gesetzmäßigkeiten über die relative Lage der beiden Flüssigkeitspunkte in bezug auf den Dampfpunkt werden nach $\mathrm{S} \mathrm{chr}$ e in e m a k e r s hergeleitet. Es wird ferner bewiesen, daß ein Maximum oder Minimum der Temperatur bzw. des Druckes im heterogenen Gebiet (Dreiphasenkoexistenzgebiet) dann und nur dann auftritt, wenn entweder die Konzentration einer Komponente Null ist (binäres System), oder die Darstellungspunkte der beiden Flüssigkeiten und des Dampfes auf einer Geraden liegen, oder ein kritischer Punkt vorliegt. Der Begriff und die Bedeutung der Destillationslinien für heterogene ternäre Flüssigkeitsgemische werden exakt formuliert. Es wird gezeigt, daß bei Rektifikation dieser Gemische Sinken des Siedepunktes im Rückstande möglich ist. Die Siedeflächen werden diskutiert. Folgende Sätze werden gewonnen: 1. Ein Minimum des Siedepunkts im heterogenen Gebiet kann nur dann der tiefste Siedepunkt des gesamten ternären Systems sein, wenn die Dampfzusammensetzung zwischen den Zusammensetzungen der beiden flüssigen Schichten liegt. 2. Ein Maximum des Siedepunkts im heterogenen Gebiet kann nie der höchste Siedepunkt des gesamten ternären Systems sein. - Es wird darauf hingewiesen, daß der Begriff „ternärer azeotroper Punkt“ nur auf Siedepunkts- bzw. Dampfdruckextrema im homogenen Gebiet (d.h. auf wirkliche Extrema in den Siedeflächen) angewendet werden sollte. - Anhangsweise werden schließlich die allgemeinsten Stabilitätskriterien aus den Gibbsschen Gleichungen abgeleitet; es wird insbesondere die Äquivalenz der für die totalen und der für die mittleren molaren charakteristischen Funktionen formulierten Stabilitätsbedingungen mathematisch bewiesen.
\end{abstract}

$\mathrm{I}$ $\mathrm{n}$ früheren Abhandlungen sind binäre Systeme ${ }^{1}$ und ternäre Systeme ohne Mischungslücke ${ }^{2,3}$ in bezug auf die Verdampfungsgleichgewichte thermodynamisch untersucht worden. Gegenstand der vorliegenden Arbeit sind ternäre Flüssigkeitsgemische, die in bestimmten Konzentrationsbereichen Entmischung zeigen, so daß zwei flüssige Schichten auftreten. Sind Druck und Temperatur festgelegt, so gibt es in diesem Falle eine ganze Reihe von Flüssigkeiten, von denen je zwei koexistent sind. Bei gegebener Temperatur können ferner ternäre Flüssigkeitspaare mit Dämpfen bestimmter Zusammensetzung im Gleichgewicht sein, wobei jedem Phasentripel (d. h. jeder Kombination der drei koexistenten Phasen) ein bestimmter Dampfdruck entspricht; bei gegebenem

1 IV. Mitt.: R. H a a s e, Z. physik. Chem. (im Druck).

2 V. Mitt.: R. H a a s e, Z. physik. Chem. (im Druck).

${ }^{3}$ R. H a a s e, Z. Naturforschg. 4a, 342 [1949].
Druck hat demgemäß jedes Phasentripel eine bestimmte Siedetemperatur. Schließlich ist es auch möglich, daß drei flüssige Schichten auftreten und im Gleichgewicht mit Dampf sind. Bei gegebener Temperatur liegen dann der Dampfdruck und die $\mathrm{Zu}$ sammensetzungen aller vier Phasen fest. Derartige Dreistoffsysteme mit drei koexistenten Flüssigkeiten sind von Schreinemakers ${ }^{4}$ so vollständig behandelt worden, daß wir uns in dieser Darstellung auf die Untersuchung der ternären Systeme mit zwei flüssigen Phasen beschränken können. Hier geht eine

${ }^{4}$ F. A. H. S chrein e makers, Z. physik. Chem. 35, 459 [1900]; 36, 710 [1901]; 37, 129 [1901]; 38, 227 [1901]; 39, 485 [1902]; 40, 440 [1902]; 41, 331 [1902]. Zusammenfass., aber nicht vollständige Darstellung bei J. P. K u e n e n, Theorie der Verdampfung und Verflüssigung von Gemischen, S. 226, Leipzig 1906. - Über Systeme ohne Dampf vgl. H. W. B a khu is R o o z e b o o m, Die heterogenen Gleichgewichte, 3. Heft, 2. Teil, Braunschweig 1913. 
Reihe wichtiger Erkenntnisse ebenfalls auf S chrei n e makers zurück, der auch die ersten systematischen Messungen durchführte. Einige allgemeine Gesetzmäßigkeiten für Gleichgewichte von mehreren Flüssigkeiten mit Dampf bei Systemen mit beliebig vielen Komponenten wurden in einer früheren Arbeit ${ }^{5}$ hergeleitet.

Gleichgewichte zweier Flüssigkeiten (Entmischungskurve und kritischer Entmischungspunkt) werden im ersten Kapitel, Gleichgewichte zweier Flüssigkeiten mit Dampf im zweiten Kapitel behandelt. Die Siedeflächen eines ternären Systems mit Mischungslücke untersuchen wir im dritten Kapitel. Allgemeine Ausführungen über Stabilitätsbedingungen sind der Inhalt eines Anhangs.

Die früheren Mitteilungen dieser ${ }^{1,2,5-7}$ Reihe werden als I, II, III, IV und V, eine Abhandlung über Zustandsfunktionen bei Vielkomponentensystemen ${ }^{8}$ als $\mathrm{A}$ und eine Arbeit über die Theorie der Destillationslinien ${ }^{3}$ als B zitiert.

1. Entmischungskurve und kritischer Punkt

$x_{1}$ und $x_{2}$ seien die Molenbrüche der Komponenten 1 und 2 in einer ternären flüssigen Mischung beim Druck $P$ und der Temperatur T. Die Freie Enthalpie (Gibbsche Freie Energie), das Volumen und die Entropie, jeweils auf $1 \mathrm{Mol}$ Mischung bezogen, seien mit $G$, $V$ und $S$ bezeichnet. Aus den thermodynamischen Beziehungen

$$
\partial G / \partial P=V, \quad(1) \quad \partial G / \partial T=-S
$$

sowie aus den Stabilitätsbedingungen [s. II (1), V (13), B (1)]:

$$
\left|\begin{array}{lr}
\frac{\partial^{2} G}{\partial x_{1}{ }^{2}} & \frac{\partial^{2} G}{\partial x_{1} \partial x_{2}} \\
\frac{\partial^{2} G}{\partial x_{2} \partial x_{1}} & \frac{\partial^{2} G}{\partial x_{2}{ }^{2}}
\end{array}\right|>0, \quad \text { (3 a) } \begin{aligned}
& \frac{\partial^{2} G}{\partial x_{1}{ }^{2}}>0 \\
& \frac{\partial^{2} G}{\partial x_{2}{ }^{2}}>0
\end{aligned}
$$

ergibt sich, daß die für bestimmte Werte von $P$ und $T$ konstruierte $G\left(x_{1}, x_{2}\right)$-Fläche für ternäre Gemische eine analoge Bedeutung hat wie für binäre Gemische die $G(x)$-Kurve ${ }^{1}$. Wir denken uns die Variablen $x_{1}$ und $x_{2}$ in Möbiusschen Dreieckskoordinaten (im Gibbs schen Darstellungsdreieck) und die zugehörigen $G$ als senkrecht auf der Dreiecksebene errichtete Ordinaten abgebildet. Durch die Betrachtung der $G$ $\left(x_{1}, x_{2}\right)$-Fläche und ihrer Veränderung mit Druck und Temperatur können wir allgemeine Erkenntnisse über Stabilität und Koexistenzen von Phasen gewinnen ${ }^{9}$.

"III. Mitt.: R. H a a s e, Z. Naturforschg. 3 a, 323 [1948].

${ }^{6}$ I. Mitt.: W. J o s t, Z. Naturforschg. 1, 576 [1946].

‘ II. Mitt.: R. H a s e, Z. Naturforschg. 2a, 492 [1947].

R. Ha a se, Z. Naturforschg. 3 a, 285 [1948].
Die Beziehungen (3) bedeuten, daß bei stabilen Phasen die $G\left(x_{1}, x_{2}\right)$-Fläche konvex-konvex in bezug auf die Dreiecksebene ist. Treten labile Gebiete auf, weil eine der Bedingungen (3) nicht erfüllt ist, so weist die G-Fläche konvex-konkave Teile auf, so daß in der Fläche „Falten“ entstehen. Die Grenze zwischen Stabilität und Labilität, also dem konvexkonvexen und dem konvex-konkaven Gebiet, bildet eine Kurve, für die gilt:

$$
D=\frac{\partial^{2} G}{\partial x_{1}{ }^{2}} \frac{\partial^{2} G}{\partial x_{2}{ }^{2}}-\left(\frac{\partial^{2} G}{\partial x_{1} \partial x_{2}}\right)^{2}=0,
$$

wobei die Ungleichungen (3b) und (3c) weiterhin gültig sind. Diese Kurve heißt „Spinodalkurve“. Erst innerhalb des von der Spinodalkurve umgrenzten Gebietes verlaufen die Kurven $\delta^{2} G / \delta x_{1}{ }^{2}=0$ und $\delta^{2} G / \delta x_{2}{ }^{2}=0$. Eine notwendige Folge der Faltenform der Fläche ist die Tatsache, daß sich stets Tangentialebenen angeben lassen, die die Fläche in zwei Punkten berühren und deshalb „Doppelberührungsebenen" genannt werden. Bezeichnen wir zwei Punkte der Fläche mit $G^{\prime}\left(x_{1}{ }^{\prime}, x_{2}{ }^{\prime}\right)$ und $G^{\prime \prime}\left(x_{1}{ }^{\prime \prime}\right.$, $\left.x_{2}{ }^{\prime \prime}\right)$, so gibt offensichtlich das Gleichungssystem

$$
\begin{aligned}
& \left(\frac{\partial G}{\partial x_{1}}\right)^{\prime}=\left(\frac{\partial G}{\partial x_{1}}\right)^{\prime \prime}, \quad \text { (5 a) } \quad\left(\frac{\partial G}{\partial x_{2}}\right)^{\prime}=\left(\frac{\partial G}{\partial x_{2}}\right)^{\prime \prime}, \quad(5 \mathrm{~b}) \\
& \left(G-x_{1} \frac{\partial G}{\partial x_{1}}-x_{2} \frac{\partial G}{\partial x_{2}}\right)^{\prime}=\left(G-x_{1} \frac{\partial G}{\partial x_{1}}-x_{2} \frac{\partial G}{\partial x_{2}}\right)^{\prime \prime}
\end{aligned}
$$

die Bedingung dafür an, daß die betrachteten Punkte Berührungspunkte einer Doppelberührungsebene sind. Die Kurve, auf der diese Berührungspunkte liegen, und ebenso ihre Projektion auf die Dreiecksebene wird „Binodalkurve“ oder „Konnodalkurve“ genannt. Die Geraden, die je zwei Berührungspunkte verbinden, und ihre Projektionen auf die Dreiecksebene heißen „Konnoden“. Sie sind die Erzeugenden der Regelfläche, die durch Abrollen der Doppelberührungsebene auf der G-Fläche entsteht. Wenn diese Ebene sich der Fläche entlang bewegt, kann der Fall eintreten, daß die beiden Berührungspunkte zusammenfallen. Der Punkt der Fläche, an dem dieses Zusammenfallen stattfindet, heißt - ebenso wie seine Projektion auf die Dreiecksebene — „Faltenpunkt“. Es läßt sich zeigen, daß die Binodalkurve und die

${ }^{9}$ s. z. B. H. W. Bakhuis Roozeboom ${ }^{4}$, S. 284 . Die mathematischen Grundlagen schuf D. J. Korteweg in seinen Arbeiten über die „Faltentheorie“: S.-B. Akad. Wiss. Wien, Abt. II a 98 [1889]; Arch. néerl. Sci. exact. natur. 24, 57, 295 [1891], (II) 8, 235 [1903]. 
Spinodalkurve einander im Faltenpunkt berühren und daß im einfachsten Falle - bei Ausschluß von Knikken, Spitzen usw. in der Fläche sowie von sog. Doppelfaltenpunkten - die Spinodalkurve innerhalb der Konnodalkurve verläuft. Für unsere Betrachtungen kommt nur dieser einfachste Fall in Frage, der in Abb. 1 wiedergegeben ist. Es ist die G-Fläche - der Anschaulichkeit halber hier perspektivisch in rechtwinkligen Koordinaten - in der Umgebung eines Faltenpunktes $\mathrm{K}$ gezeichnet. OKP ist die Binodalkurve, QKR die Spinodalkurve. Die Schnittkurve AOPB, die durch eine zur $x_{1}$-Achse parallele Vertikalebene auf der Fläche erzeugt wird, weist zwei Wendepunkte auf, für welche gilt: $\delta^{2} G / \delta x_{1}{ }^{2}=0$. Nach

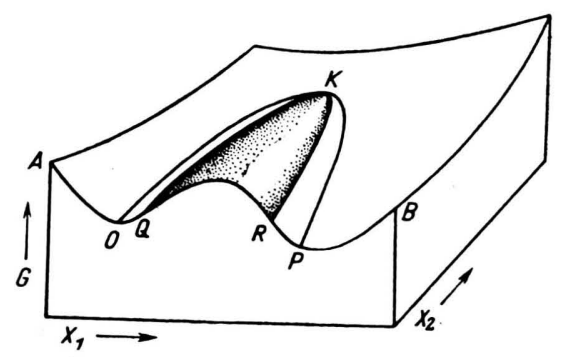

Abb. 1.

Obigem liegen diese beiden Punkte zwischen $\mathrm{Q}$ und R. Wenn aber die genannte Schnittkurve gerade in der Grenzebene $x_{2}=0$ liegt, so fallen die Wendepunkte mit $\mathrm{Q}$ und $\mathrm{R}$ zusammen.

Welche physikalische Bedeutung haben Binodalkurve und Faltenpunkt? Betrachten wir die Gln. (5). Sie geben nichts anderes als die Koexistenzbedingungen für zwei ternäre Phasen an [s. A, Gln. (19)]; denn sie sind äquivalent den Gleichungen $\mu_{1}{ }^{\prime}=\mu_{1}^{\prime \prime}$, $\mu_{2}^{\prime}=\mu_{2}^{\prime \prime}, \mu_{3}^{\prime}=\mu_{3}^{\prime \prime}$, worin $\mu_{i}$ das chemische Potential der Komponente $i$ bedeutet. Die Endpunkte der Konnoden entsprechen also je zwei koexistenten Phasen, und die Binodalkurve ist die Entmischungskurve (Löslichkeitskurve) für gegebene Werte von $P$ und $T$. Der Faltenpunkt schließlich entspricht dem kritischen Entmischungspunkt, da eine kritische Phase immer dadurch gekennzeichnet ist, daß sie durch $\mathrm{Zu}$ sammenfallen zweier koexistenter Phasen einerseits und des stabilen und des labilen Gebietes andererseits entsteht ${ }^{10}$, wie zuerst $\mathrm{G}$ i b b s ${ }^{11}$ darlegte.

10 Wir sehen in dieser orientierenden Darstellung von den Komplikationen ab, die sich daraus ergeben könnten, daß nach J. E. Mayer u. Mitarbb. am kritischen Entmischungspunkt eventuell anomale Phasenumwandlungen vorliegen.

11 J. W. Gi b b s, Scientific Papers I, S. 99, 105, 109, 128, 129. London, New York u. Bombay 1906.
Das Gebiet zwischen Binodal- und Spinodalkurve entspricht, gemäß den Anschauungen von v a n d e r W a a ls, den metastabilen Zuständen, das Gebiet innerhalb der Spinodalkurve den absolut instabilen (labilen) Zuständen ${ }^{12}$.

Wenn $P$ und $T$ gegeben sind, muß ein kritischer Punkt in einem ternären System durch bestimmte Werte von $x_{1}$ und $x_{2}$ charakterisiert sein. Wir benötigen zur Auffindung dieser Werte außer (4) noch eine zweite Gleichung. Diese erhalten wir folgendermaßen ${ }^{11}$ :

Wir betrachten eine kritische Phase, die aus $m_{1}$ Molen der Komponente 1, $m_{2}$ Molen der Komponente 2 und $m_{3}$ Molen der Komponente 3 besteht. Da Änderungen der Gesamtmasse nicht interessieren, setzen wir $m_{3}=$ const. Die (totale) Freie Enthalpie der Phase sei g. Dann gilt:

$$
\mu_{i}=\partial g / \partial m_{i} .
$$

Anstelle von Gl. (4) erhalten wir als erste Bedingungsgleichung für den kritischen Punkt ${ }^{13}$ :

$$
D^{\prime}=\frac{\partial^{2} g}{\partial m_{1}{ }^{2}} \frac{\partial^{2} g}{\partial m_{2}{ }^{2}}-\left(\frac{\partial^{2} g}{\partial m_{1}} \frac{\partial m_{2}}{\partial}\right)^{2}=0 .
$$

Ein System von zwei koexistenten Phasen, die drei Komponenten enthalten, hat drei Freiheitsgrade. Es können daher drei der Größen $P, T, \mu_{1}, \mu_{2}$ und $\mu_{3}$ unabhängig geändert werden. Geht man von einem vorgegebenen Paar koexistenter Phasen aus, dem bestimmte Werte von $P, T, \mu_{1}, \mu_{2}$ und $\mu_{3}$ entsprechen, und hält drei dieser Größen konstant, so verläßt man bei Änderung von Druck, Temperatur oder Zusammensetzung das Koexistenzgebiet und gelangt entweder in das zwischen den beiden Phasen gelegene Gebiet metastabiler und labiler Phasen oder - bei entgegengesetztem Sinn der Änderungen - in das Gebiet stabiler Phasen. Nimmt man die entsprechende Änderung jedoch an einer kritischen Phase vor, so gelangt man stets zu einer stabilen Phase, da definitionsgemäß der kritische Punkt der Endpunkt einer

12 Auch wenn man eine Extrapolation thermodynamischer Zustandsfunktionen in das metastabile und labile Gebiet für physikalisch sinnlos hält (vgl. Anm. 1), ist die mathematische Extrapolation stets erlaubt, soweit sie nur zur Auffindung der koexistenten Phasen und der kritischen Punkte dient; lediglich die Interpretation der metastabilen Zustände (wenigstens in größerer Entfernung vom absolut stabilen Gebiet) dürfte dann nicht den obigen Vorstellungen entsprechen.

13 Wir müssen hier zunächst von der üblichen Methode der Betrachtung eines Mols Mischung abgehen, weil für das Folgende $P, T, \mu_{1}, \mu_{2}$ und $\mu_{3}$ die zweckmäßigsten Funktionen sind. Über die Äquivalenz der Gln. (4) u. (7) s. Anhang. 
Reihe von Paaren koexistenter Phasen ist und gleichzeitig an der Stabilitätsgrenze liegt. Eine kritische Phase kann also nicht instabil werden, wenn drei der Größen $P, T, \mu_{1}, \mu_{2}$ und $\mu_{3}$ bei irgendeiner Änderung konstant bleiben. Daraus folgt, daß für $d P=0$, $d T=0, d \mu_{1}=0$ die Änderung von $D^{\prime}$ [s. Gl. (7)] nicht negativ sein kann. Der Ausdruck

$$
d D^{\prime}=\frac{\partial D^{\prime}}{\partial m_{1}} d m_{1}+\frac{\partial D^{\prime}}{\partial m_{2}} d m_{2}
$$

kann aber auch nicht positiv sein, da sonst bei Vorzeichenwechsel von $d m_{1}$ und $d m_{2}$ gelten würde: $d D^{\prime}<0$.

Daher ergibt sich für konstanten Druck und konstante Temperatur folgendes Gleichungssystem:

$$
\begin{aligned}
& d \mu_{1}=\frac{\partial \mu_{1}}{\partial m_{1}} d m_{1}+\frac{\partial \mu_{1}}{\partial m_{2}} d m_{2}=0, \\
& d D^{\prime}=\frac{\partial D^{\prime}}{\partial m_{1}} d m_{1}+\frac{\partial D^{\prime}}{\partial m_{2}} d m_{2}=0 .
\end{aligned}
$$

Hieraus folgt mit (6):

$$
\left|\begin{array}{cc}
\frac{\partial^{2} g}{\partial m_{1}{ }^{2}} & \frac{\partial^{2} g}{\partial m_{1} \partial m_{2}} \\
\frac{\partial D^{\prime}}{\partial m_{1}} & \frac{\partial D^{\prime}}{\partial m_{2}}
\end{array}\right|=0 .
$$

(7) und (8) sind die beiden Bedingungsgleichungen für den kritischen Punkt.

Beziehen wir alle Größen jetzt wieder auf ein $\mathrm{Mol}$ Mischung, so erhalten wir mit (4) anstelle von (7) und (8):

$$
\begin{aligned}
D= & \frac{\partial^{2} G}{\partial x_{1}{ }^{2}} \frac{\partial^{2} G}{\partial x_{2}{ }^{2}}-\left(\frac{\partial^{2} G}{\partial x_{1} \partial x_{2}}\right)^{2}=0, \\
& \frac{\partial^{2} G}{\partial x_{1}{ }^{2}} \frac{\partial D}{\partial x_{2}}-\frac{\partial^{2} G}{\partial x_{1} \partial x_{2}} \frac{\partial D}{\partial x_{1}}=0 .
\end{aligned}
$$

Ist $G$ als Funktion von $x_{1}$ und $x_{2}$ bekannt, so lassen sich hieraus die kritischen Konzentrationen errechnen.

Auf Grund der Gültigkeit der universellen Grenzgesetze für unendliche Verdünnung können wir für eine beliebige ternäre Mischung schreiben $(R=$ Gaskonstante, $\Delta G E=\mathrm{zu}-$ sätzliche molare Freie Enthalpie):

$$
\begin{aligned}
& \frac{\partial^{2} G}{\partial x_{1}{ }^{2}}=R T\left[\frac{1-x_{2}}{x_{1}\left(1-x_{1}-x_{2}\right)}+\beta\right], \\
& \frac{\partial^{2} G}{\partial x_{2}{ }^{2}}=R T\left[\frac{1-x_{1}}{x_{2}\left(1-x_{1}-x_{2}\right)}+\gamma\right], \\
& \frac{\partial^{2} G}{\partial x_{1} \partial x_{2}}=R T\left[\frac{1}{1-x_{1}-x_{2}}+\delta\right],
\end{aligned}
$$

worin

$$
\begin{gathered}
R T \beta=\frac{\partial^{2}}{\partial x_{1}{ }^{2}} \Delta G^{E} . \\
R T \gamma=\frac{\partial^{2}}{\partial x_{2}{ }^{2}} \Delta G^{E}, \\
R T \delta=\frac{\partial^{2}}{\partial x_{1}} \frac{\partial x_{2}}{R} \Delta G^{E}
\end{gathered}
$$

bedeuten. (9a) und (Gln. $a-c)$ ergeben die Gleichung der Spinodalkurve:

$$
\begin{gathered}
1+\beta x_{1}\left(1-x_{1}\right)+\gamma x_{2}\left(1-x_{2}\right)-2 \delta x_{1} x_{2} \\
+\left(\beta \gamma-\delta^{2}\right) x_{1} x_{2}\left(1-x_{1}-x_{2}\right)=0 .
\end{gathered}
$$

Bei Kenntnis von $\triangle G^{E}\left(x_{1}, x_{2}\right)$ aus der statistischen Theorie oder aus Dampfdruckmessungen kann mit (Gl. d) entschieden werden, ob bei den vorgegebenen Werten von $T$ und $P$ Entmischung eintritt. Verläuft nämlich die Spinodalkurve teilweise oder ganz innerhalb des Darstellungsdreiecks, so muß das ternäre System in gewissen Konzentrationsbereichen in zwei Flüssigkeiten zerfallen. (Gl. d) ist gleichzeitig die allgemeine Form der ersten Gleichung für den kritischen Entmischungspunkt; die zweite (sehr verwickelte) Gleichung erhält man aus (9b) und $(\mathrm{Gln} . \mathrm{a}-\mathrm{c})$.

Am einfachsten sind die Verhältnisse, wenn $\beta, \gamma$ und $\delta$ unabhängig von der Zusammensetzung sind, wie z. B. bei dem symmetrischen Ansatz [vgl. I (10)]:

$$
\begin{gathered}
\Delta G^{E}=A_{12} x_{1} x_{2}+A_{13} x_{1} x_{3}+A_{23} x_{2} x_{3}, \\
\left(x_{3}=1-x_{1}-x_{2}\right),
\end{gathered}
$$

worin die $A_{i k}$ nur von der Temperatur und der Art der Komponenten $i$ und $k$ abhängen. Für diesen Ansatz folgt:

$$
\begin{gathered}
\beta=-\frac{2 A_{13}}{R T} \equiv-2 B_{13}, \quad \gamma=-\frac{2 A_{23}}{R T} \equiv-2 B_{23} . \\
\delta=\frac{A_{12}-A_{13}-A_{23}}{R T} \equiv B_{12}-B_{13}-B_{23} . \quad \text { Gl. }
\end{gathered}
$$

(Gl. d) und (Gl.f) ergeben:

$$
\begin{gathered}
1-2\left(B_{12} x_{1} x_{2}+B_{13} x_{1} x_{3}+B_{23} x_{22} x_{3}\right) \\
+\left(2 B_{12} B_{13}+2 B_{12} B_{23}+2 B_{13} B_{93}-B^{2}{ }_{12}-B^{2}{ }_{13}-B^{2}{ }_{23}\right) \\
\cdot x_{1} x_{2} x_{3}=0 .
\end{gathered}
$$

Die zweite Gleichung für den kritischen Entmischungspunkt ist auch bei Voraussetzung des speziellen Ansatzes (Gl. e) noch sehr kompliziert.

$\mathrm{S} \operatorname{cott}^{13 \mathrm{a}}$ und $\mathrm{Tompa}{ }^{13 b}$ diskutierten entsprechende Beziehungen für ternäre Gemische mit einem oder zwei Hochpolymeren. Sie stellten die Mischungswärme durch einen in den Volumenbrüchen $\varphi_{i}$ symmetrischen Ausdruck und die Mischungsentropie durch die Flory-Huggins sche Näherung dar. Ihre Gleichungen für den Faltenpunkt bei Systemen mit einer hochmolekularen Komponente gehen formal in die für den Ansatz (Gl. e) gültigen über,

13 a R. L. S c o t t, J. Chem. Physics 17, 268, 279 [1949]. $13 \mathrm{~b}$ H. To mpa, J. Chem. Physics 17, 1003, 1006 [1949]. 
wenn man in ihren Formeln $\mu_{i k}=B_{i k}, \varphi_{i}=x_{i}$ und $m$ (Polymerisationsgrad) $=1$ setzt. Dies beruht auf der mathematischen Gestalt von (Gl. e) und bedeutet nicht, daß sie ein Sonderfall der von S cott und $\mathrm{T}$ o $\mathrm{mpa}$ benutzten Näherung ist. (Gl. e) stellt vielmehr die auf ternäre Gemische sinngemäß erweiterte Formulierung einer empirischen Näherung dar, die für zahlreiche binäre niedrigmolekulare Nichtelektrolytlösungen gilt und die weder ideale Mischungsentropie noch Gleichheit der Molvolumina der Komponenten voraussetzt ${ }^{1}$.

Prigogin e ${ }^{13 c}$ untersuchte theoretisch den Einfluß geringer Mengen einer dritten Komponente auf die kritische Entmischungstemperatur eines binären Systems. Er ging dabei von eìnem Ansatz derselben Form wie (Gl. e) aus, setzte aber temperaturunabhängige Werte der $A_{i k}$, d. h. reguläre Mischungen mit in den $x_{i}$ symmetrischen Verlauf der Mischungswärme voraus.

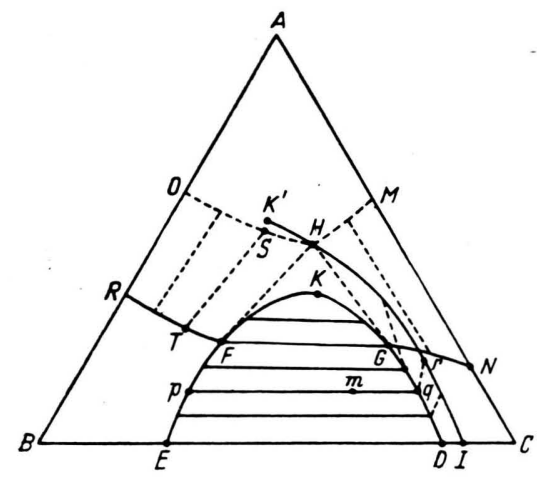

Abb. 2.

Die Gleichung für die Binodalkurve endlich läßt sich nur mit weiteren Vereinfachungen diskutieren. Für Systeme mit Hochpolymeren wurde dies von $\mathrm{Scott}{ }^{13} \mathrm{a}$ und $\mathrm{M}$ ü n s t e r ${ }^{13} \mathrm{~d}$ durchgeführt.

Es sei angemerkt, daß die entsprechenden Gleichungen füi binäre Systeme, in denen es nur einen unabhängigen Molenbruch $x$ gibt, lauten ${ }^{13} \mathrm{e}: \partial^{2} G / \partial x^{2}=0, \quad \partial^{3} G / \partial x^{3}=0$. Diese Beziehungen erlauben bei einfachen Ansätzen für $G$ sofort die Berechnung. der kritischen Konzentration und der kritischen Entmischungstemperatur.

In Abb. 2 ist eine Entmischungskurve EFKGD gezeichnet, die einen kritischen Punkt $\mathrm{K}$ aufweist. Die ausgezogenen Geraden sind die Konnoden; F und G sind z. B. koexistente flüssige Phasen. Es gibt auch Fälle, in denen ein geschlossenes Entmischungsgebiet

$13 \mathrm{c}$ I. Prigogine, Bull. Soc. chim. Belgique 52, 115 [1943].

${ }_{13}$ d A. M ü n s te r, J. Polymer Sci. (im Druck).

13 e Diese Gleichungen sind wegen des aus (31) und aus der Gibbs-Duhem-Margulesschen Beziehung folgenden Zusammenhangs

$$
(1-x)\left(\partial^{2} G / \partial x^{2}\right)=\partial \mu_{1} / \partial x
$$

vollkommen gleichwertig den Bedingungen

$$
\partial \mu_{1} / \partial x=0, \quad \partial^{2} \mu_{1} / \partial x^{2}=0 .
$$

mit zwei kritischen Punkten oder ein von einer Dreiecksseite zu einer anderen verlaufendes Entmischungsgebiet ohne kritischen Punkt auftritt. Schließlich können von zwei oder drei Dreiecksseiten je eine Löslichkeitskurve wie in Abb. 2 ausgehen, die entweder getrennt bleiben oder ineinander übergehen, wobei sich auch Systeme mit drei flüssigen Phasen bilden können. Durch Druck- und besonders durch Temperaturänderung lassen sich die verschiedenen Typen ineinander überführen. Eine ausführliche Darstellung der möglichen Fälle hat $\mathrm{S} \mathrm{c} \mathrm{h} \mathrm{r} \mathrm{e} \mathrm{i} \mathrm{n} \mathrm{e} \mathrm{m} \mathrm{a} \mathrm{k} \mathrm{e} \mathrm{r} \mathrm{s}{ }^{14}$ gegeben. Später wurden diese Untersuchungen durch Messungen von $\mathrm{Hill}{ }^{15}$ ergänzt. Der in Abb. 2 dargestellte Typ der Löslichkeitskurve ist der häufigste.

Über die experimentelle Ermittlung und einige Anwendungen der Entmischungsdiagramme wurde an anderer Stelle ${ }^{16}$ berichtet.

\section{Gleichgewichte zweier Flüssig- keiten mit Dampf}

Ist der Druck hinreichend hoch bzw. die Temperatur hinreichend niedrig, so ist die Gasphase gegenüber flüssigen Phasen nicht stabil ${ }^{*}$. Dies gibt sich in dem von uns betrachteten Falle ternärer Systeme mit zwei Flüssigkeiten in der geometrischen Darstellung dadurch zu erkennen, daß die stets konvex-konvexe G-F'läche der Gasphase, genannt das „Dampfblatt“, ganz obcrhalb der G-Fläche der Flüssigkcit liegt; diese letzte Fläche, die wir bisher allein betrachtet haben, sei kurz als „Flüssigkeitsblatt“ bezeichnet. Durch Erniedrigung des Druckes bzw. Erhöhung der Temperatur bewegt sich nach Gl. (1) bzw. (2) das Dampfblatt in Richtung des Flüssigkeitsblattes; denn jeder Punkt des Dampfblattes verschiebt sich schneller nach unten als der entsprechende Punkt des Flüssigkeitsblattes, weil Volumen und Entropie bei jeder beliebigen Zusammensetzung für den Dampf größere Werte haben als für die Flüssigkeit. Dadurch wird es schließlich zur Durchschneidung beider Blätter kommen, so daß an das Flüssigkeitsblatt und das Dampfblatt eine Berührungsebene gelegt werden kann, die drei Berührungspunkte aufweist; diese dreifache Berührungsebene, deren Lage sich bei Druck- oder Temperaturänderung verschiebt, entspricht einem

${ }^{14}$ H. W. Bakhuis Roozeboom ${ }^{4}$, S. 1 ff. F. A. H. S chreinem a kers, Z. physik. Chem. 25, 545 [1898]; 29, 586 [1899]; 33, 84 [1900].

15 A. E. Hill, J. Amer. chem. Soc. 44, 1163, 1186 [1922]; Hill u. Miller, ebenda 47, 2702 [1925].

10 R. H a a s e, Angew. Chem., Teil A 60, 4 [1948].

* In diesem Kapitel werden auch metastabile Phasen als ,nicht stabil“ bezeichnet. 
Gleichgewicht von drei Phasen (zwei Flüssigkeiten und Dampf). Jedes dieser Dreiphasensysteme hat also bei konstantem Druck eine bestimmte Siedetemperatur und bei konstanter Temperatur einen bestimmten Dampfdruck.

Die Entmischungskurve in Abb. 2 ist keine Binodalkurve mehr, da diese für konstanten Druck und konstante Temperatur definiert ist; bei konstanter Temperatur wird jedoch die Binodalkurve nur sehr wenig von einer Entmischungskurve, bei der jedes Phasentripel zu einem bestimmten Dampfdruck gehört, abweichen, da der Einfluß des Druckes auf Gleichgewichte kondensierter Phasen gering ist; die $\mathrm{Ab}$ weichung ist aber sehr groß, wenn wir die Dreiphasengleichgewichte bei konstantem Druck betrachten.

Der Punkt, in dem das Dampfblatt von der dreifachen Berührungsebene berührt wird, kann nun innerhalb oder außerhalb der Binodalkurve liegen. Dementsprechend liegt die Zusammensetzung des mit zwei flüssigen Phasen koexistenten Dampfes zwischen den Flüssigkeitszusammensetzungen oder außerhalb derselben. Die Projektion der Kurve, welche die Gleichgewichtsdämpfe angibt, auf die Dreiecksebene nennen wir „Dampfkurve“; diese kann nach Obigem innerhalb (Abb. 4, 5, 7) oder außerhalb (Abb. 2, 3, 6), oder auch teils innerhalb und teils außerhalb der Entmischungskurve verlaufen.

Betrachtet man nicht nur die dreifachen, sondern auch die Doppelberührungsebenen, so erhält man für jeden Druck und jede Temperatur alle stabilen Gleichgewichte des ternären Systems, also auch solche zwischen zwei Flüssigkeiten (mithin den bei den betreffenden Werten von $P$ und $T$ stabilen Teil der Binodalkurve) und solche zwischen einer Flüssigkeit und Dampf (mithin die Verdampfungs- und Kondensationskurven bei den betreffenden Werten von $P$ und $T)$. Schreinemakers ${ }^{4}$ hat die hierbei sich ergebenden Möglichkeiten sorgfältig analysiert. R e in ders und de Minje $\mathrm{r}^{17}$ haben neuerdings an einigen interessanten Systemen experimentelle Untersuchungen durchgeführt.

In Abb. 2 ist einer der zahlreichen möglichen Fälle von Gleichgewichtsdiagrammen wiedergegeben. Ein Beispiel für diesen Typ ist das von Schreinemakers ${ }^{4}$ untersuchte System Aceton (A) - Phenol (B) - Wasser (C) unterhalb $68^{\circ} \mathrm{C}$, der kritischen Entmischungstemperatur des binären Systems Phenol-Wasser. (Oberhalb dieser Temperatur löst sich das Entmischungsgebiet unter Bildung einer geschlossenen Löslichkeitskurve mit zwei kriti-

17 W. Reinders u. C. H. de Minjer, Recueil Trav. chim. Pays-Bas 66, 552, 564, 573 [1947]. schen Punkten von der Dreiecksseite BC ab.) Zwischen I und $\mathrm{C}$ liegt ein (nicht eingezeichneter) binärer azeotroper Punkt (Dampfdruckmaximum). Denken wir uns Abb. 2 auf eine bestimmte Temperatur bezogen, so gehört zu jeder der Konnoden im Entmischungsgebiet ein anderer Dampfdruck. Die Kurve IHK' ist die Dampfkurve; die von den Enden der Konnoden ausgehenden gestrichelten Geraden, z. B. qr, GH und FH, sind die Verbindungsstrecken zu den koexistenten Dämpfen. Es sind also z. B. miteinander im Gleichgewicht: die Flüssigkeiten $p$ und $q$ und der Dampf $r$, die Flüssigkeiten $F$ und $G$ und der Dampf H. Der zur kritischen Flüssigkeit gehörende Dampf ist $\mathrm{K}^{\prime}$; der zugehörige Dampfdruck ist in diesem Falle der höchste Druck im heterogenen Gebiet, während der dem binären System E+D + I entsprechende Dampfdruck der tiefste Druck aller Dreiphasensysteme ist; es läßt sich ferner ableiten, daß dieser höchste bzw. tiefste Druck ein wirkliches Maximum bzw. Minimum ist (s. unten). Längs den Dreiecksseiten BA und CA nimmt der Dampfdruck in Richtung auf A (reines Aceton) hin monoton zu. Komplizierter liegen die Verhältnisse, wenn wir längs der Seite BC fortschreiten (s. hierzu IV, Abb. 2 c): der Dampfdruck nimmt von B (reines Phenol) nach E zu, bleibt bis D als Dreiphasendruck konstant (Gleichgewichtsdampf: I), steigt dann weiter bis zu einem Punkt zwischen I und C (binäres Dampfdruckmaximum) und nimmt bis $\mathrm{C}$ (reines Wasser) wieder ab. Von den im homogenen Gebiet verlaufenden Verdampfungs- und Kondensationskurven ist ein einziges Paar eingezeichnet, nämlich dasjenige, das zum Druck des Dreiphasensystems F+G $+\mathrm{H}$ gehört: die Verdampfungskurven RF und GN mit den Kondensationskurven $\mathrm{OH}$ und HM. Die gestrichelten Geraden zwischen Verdampfungs- und Kondensationskurven, z. B. TS, FH, GH, sind die Konnoden der Gleichgewichte FlüssigkeitDampf. Die gebrochenen Kurvenzüge RFGN und OHM gelten also für den gleichen Druck; der letztgenannte Kurvenzug gibt die Dämpfe wieder, die mit den Flüssigkeiten des ersten koexistent sind. Bei demjenigen Druck, für den diese Kurvenzüge gelten, und bei derjenigen Temperatur, für die Abb. 2 gezeichnet ist, zerfällt die Dreiecksfläche in drei Teile. Im Felde AMO sind alle Gemische gasförmig, im Felde RFGNCB flüssig; die innerhalb der Binodalkurve (die jetzt, streng genommen, da sie für einen bestimmten Druck gilt, nicht mehr mit der eingezeichneten Entmischungskurve zusammenfällt) gelegenen Gemische zerfallen in zwei Flüssigkeiten; das durch $m$ dargestellte System spaltet sich z. B. in die Schichten $p$ und $q$ im Mengenverhältnis $q m: p m$. Im Felde OHMNGFR schließlich "sind nur die auf den Verdampfungskurven und der Geraden FG liegenden Flüssigkeiten im Gleichgewicht mit den Dämpfen der gebrochenen Kondensationskurve OHM möglich. Der Teil FKG der Entmischungskurve ist bei dem betrachteten Druck nicht stabil.

In Abb. 3 ist ein Stück der Entmischungskurve und der Dampfkurve in der bisherigen Darstellung, aber ohne Einzeichnung des Grunddreiecks, wiedergegeben. Die Abbildung beziehe sich auf konstanten Druck; der Pfeil gebe die Richtung steigender Temperatur an. Die Punkte $\mathrm{L}_{1}, \mathrm{~L}_{2}$ und $\mathrm{D}$ stellen die $\mathrm{Zu}$ - 


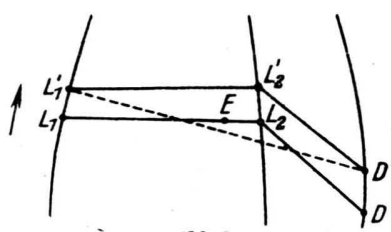

Abb.3

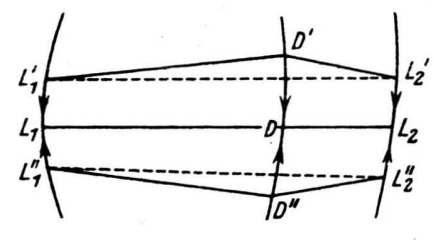

$A b b .4$

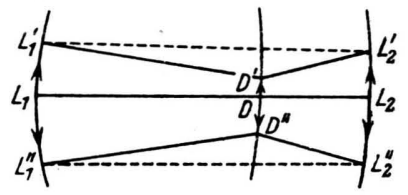

$A b b .5$ sammensetzungen zweier Flüssigkeiten und des Dampfes dar, die bei einer bestimmten (Siede-)Temperatur $T$ koexistent sind. Die drei Phasen $\mathrm{L}_{1}{ }^{\prime}, \mathrm{L}_{2}{ }^{\prime}$ und $\mathrm{D}^{\prime}$ sind demnach bei einer höheren Temperatur miteinander im Gleichgewicht.

Es kann nun bewiesen werden, daß die drei Punkte, die koexistenten Phasen entsprechen, die eingezeichnete relative Lage haben müssen. Den Beweis verdanken wir $\mathrm{S}$ chreinemakers ${ }^{4}$. Denken wir uns den Phasenkomplex $L_{1}+L_{2}$ bei der Temperatur $T$ vom koexistenten Dampf abgesperrt und erhöhen die Temperatur bei konstantem Druck beliebig wenig, so muß sich erneut Dampf bilden, der aber eine andere Zusammensetzung hat, wodurch sich die $\mathrm{Zu}$ sammensetzungen beider Flüssigkeiten ebenfalls ändern. Die drei neuen koexistenten Phasen seien $\mathrm{L}_{1}{ }^{\prime}, \mathrm{L}_{2}{ }^{\prime}$ und $\mathrm{D}^{\prime}$. Wenn nun das Mengenverhältnis der ursprünglichen Flüssigkeiten $L_{1}$ und $L_{2}$ einen solchen Wert hat, $\mathrm{da} ß$ die totale Zusammensetzung des flüssigen Phasenkomplexes durch einen Punkt $\mathrm{E}$ auf der Geraden $\mathrm{L}_{1}$ $\mathrm{L}_{2}$ dargestellt wird, so müssen die drei Punkte $\mathrm{L}_{1}{ }^{\prime}$, $\mathrm{L}_{2}{ }^{\prime}$ und $\mathrm{D}^{\prime}$ so zueinander liegen, daß $\mathrm{E}$ sich innerhalb des Dreiecks $\mathrm{L}_{1}{ }^{\prime}, \mathrm{L}_{2}{ }^{\prime}$, $\mathrm{D}^{\prime}$ befindet, da sich aus dem Komplex E die drei neuen Phasen bilden. Daraus ergibt sich, daß der Dampfpunkt auf derjenigen Seite der Verbindungsgeraden der beiden Flüssigkeiten liegt, an der bei konstantem Druck die Temperatur den niedrigeren (bzw. bei konstanter Temperatur der

* Die HHrn. Defay und Prigo gin e machten mich freundlicherweise auf folgendes aufmerksam. Der in II, V und B als Verallgemeinerung des Gibbs-Konowalowschen Gesetzes bezeichnete Satz und seine Umkehrung sowie der in III Bakhuis-Roozeboom und Schreinemakers zugeschriebene Satz sind spezielle Formen eines allgemeineren Theorems über die sog. „indifferenten Zustände“, deren Gesetzmäßigkeiten von Duhem [1899], Saurel [1900], Jouguet [1911 und 1921] und schließlich in größter Allgemeinheit von Defay [1931] untersucht wurden. Die Ableitung der verallgemeinerten ClausiusClapeyronschen Gleichung für univariante Systeme und für die indifferenten Zustände der bi- und plurivarianten Gleishgewichte geht ebenfalls auf Saurel und Defay zurück. S. hierzu R. D e f a y, Bull. Cl. Sci., Acad. roy. Belique (5) 17, 940, 1066 [1931], ferner R. D e f a y, Les extrema de tension superficielle et l'indifférence des systèmes capillaires, Brüssel 1934, und I. Pri go g i n e u. R. D ef a y, Thermodynamique chimique, Bd. II, Liège 1946.
Dampfdruck den höheren) Wert hat. Wenn wir Abb. 2 als Gleichgewichtsdiagramm für einen gegebenen Druck ansehen, so nimmt also der Siedepunkt im heterogenen Gebiet vom binären System E + D + I bis zum kritischen Punkt $\mathrm{K}$ monoton ab. Beziehen wir die Abbildungen jedoch (wie oben) auf eine bestimmte Temperatur, so nimmt der Dampfdruck von $\mathrm{E}+\mathrm{D}+\mathrm{I}$ bis $\mathrm{K}$ monoton zu.

Aus diesen Gesetzmäßigkeiten folgt ferner, daß der Dampfdruck bzw. Siedepunkt dann ein Maximum oder Minimum im Dreiphasengebiet hat, wenn die drei Punkte, die den koexistenten Phasen entsprechen, auf einer Geraden liegen. In Abb. 4 ist der Fall eines Siedepunktsmaximums, in Abb. 5 der eines Siedepunktsminimums dargestellt. Die $\mathrm{L}_{1}$ und $\mathrm{L}_{2}$ entsprechen den Flüssigkeiten und die D den Dämpfen. Die Pfeile geben wieder die Richtung steigender Temperatur an. Hier ist angenommen, daß die Dampfzusammensetzung zwischen den Flüssigkeitszusammensetzungen liegt.

Diese Maxima und Minima bei Dreiphasengleichgewichten sind ein Sonderfall der Extrema bei bivarianten Gleichgewichten ${ }^{*}$. Die früher ${ }^{5}$ angegebene allgemeine Gleichung III (12) nimmt für drei Komponenten in drei Phasen folgende Gestalt an, wenn wir die für verschiedene Phasen geltenden Größen durch die Zahl der Striche unterscheiden:

$$
\begin{aligned}
& \begin{array}{lll}
V^{\prime} & x_{1}^{\prime} & x_{2}^{\prime \prime} \\
V^{\prime \prime} & x_{1}^{\prime \prime} & x_{2}^{\prime \prime} \\
V^{\prime \prime \prime} & x_{1}^{\prime \prime \prime} & x_{2}^{\prime \prime \prime}
\end{array} \mid d P \\
& =\left|\begin{array}{lll}
S^{\prime} & x_{1}^{\prime} & x_{2}^{\prime} \\
S^{\prime \prime} & x_{1}^{\prime \prime} & x_{2}{ }^{\prime \prime} \\
S^{\prime \prime \prime} & x_{1}^{\prime \prime \prime} & x_{2}{ }^{\prime \prime}
\end{array}\right| d T+\left|\begin{array}{lll}
1 & x_{1}^{\prime} & x_{2}^{\prime} \\
1 & x_{1}^{\prime \prime} & x_{2}^{\prime \prime} \\
1 & x_{1}^{\prime \prime \prime} & x_{2}^{\prime \prime \prime}
\end{array}\right| d \mu_{3},
\end{aligned}
$$

oder abgekürzt

$$
A d P=B d T+C d u_{3} .
$$

Damit ein Maximum oder Minimum des Druckes bei konstanter Temperatur bzw. der Temperatur bei konstantem Druck auftritt, muß gelten:

$$
A d P=B d^{\prime} T \text {. }
$$


Die hierfür notwendige und hinreichende Bedingung

$$
C=0
$$

soll, obwohl früher ${ }^{5}$ allgemein diskutiert, für den vorliegenden Fall noch einmal genauer betrachtet werden.

(13) gilt, wènn die $x_{i}$ solche Werte haben, daß eine der folgenden Bedingungen erfüllt ist (die Möglichkeit, daß gewisse $x_{i}$ entweder 0 oder 1 werden, schließen wir hier aus, da wir feste Phasen nicht in Betracht ziehen):

I. $\quad \alpha_{1} x_{1}{ }^{\prime}+\alpha_{2} x_{1}{ }^{\prime \prime}=x_{1}{ }^{\prime \prime}$,

$$
\begin{aligned}
\alpha_{1} x_{2}{ }^{\prime}+\alpha_{2} x_{2}{ }^{\prime \prime} & =x_{2}{ }^{\prime \prime}, \\
\alpha_{1}+\alpha_{2} & =1 .
\end{aligned}
$$

Sonderfall:

I a. $\alpha_{1}=0, \alpha_{2}=1\left(\right.$ oder $\left.\alpha_{1}=1, \alpha_{2}=0\right)$.

Es folgt:

$$
\begin{aligned}
& x_{1}{ }^{\prime \prime}=x_{1}{ }^{\prime \prime \prime} \\
& x_{2}{ }^{\prime \prime}=x_{2}{ }^{\prime \prime}
\end{aligned} \quad\left(\text { oder: } \begin{array}{l}
x_{1}{ }^{\prime}=x_{1}{ }^{\prime \prime \prime} \\
x_{2}{ }^{\prime}=x_{2}{ }^{\prime \prime}
\end{array}\right) .
$$

(Eine dritte Möglichkeit ergibt sich durch Vertauschung des Indices, da die dritte Phase ('") willkürlich gewählt wurde).

II. $\beta_{1} x_{1}{ }^{\prime}+\beta_{2} x_{2}{ }^{\prime}=1$,

$\beta_{1} x_{1}^{\prime \prime}+\beta_{2} x_{2}^{\prime \prime}=1$,

$\beta_{1} x_{1}{ }^{\prime \prime}+\beta_{2} x_{2}{ }^{\prime \prime \prime}=1$.

Sonderfall:

$$
\text { II a. } \begin{array}{ll} 
& \beta_{1}=\beta_{2}=1 . \quad \text { Es folgt: } \\
& x_{1}^{\prime}+x_{2}^{\prime}=1, \\
& x_{1}^{\prime \prime}+x_{2}^{\prime \prime}=1, \\
& x_{1}^{\prime \prime \prime}+x_{2}^{\prime \prime \prime}=1 .
\end{array}
$$

Fall I, der schon von Gib b s ${ }^{11}$ diskutiert wurde, entspricht geometrisch der Bedingung, daß der Punkt $\mathrm{P}^{\prime \prime \prime}\left(x_{1}{ }^{\prime \prime \prime}, x_{2}{ }^{\prime \prime \prime}\right)$ im Darstellungsdreieck auf der Verbindungsgeraden der Punkte $\mathrm{P}^{\prime}\left(x_{1}{ }^{\prime}, x_{2}{ }^{\prime}\right)$ und $\mathrm{P}^{\prime \prime}$ $\left(x_{1}^{\prime \prime}, x_{2}{ }^{\prime \prime}\right)$ liegt, wobei gilt: $\overline{\mathrm{P}^{\prime} \mathrm{P}^{\prime \prime \prime}}: \overline{\mathrm{P}^{\prime \prime} \mathrm{P}^{\prime \prime \prime}}=\alpha_{2}: \alpha_{1}$. Dies bedeutet physikalisch die Möglichkeit des Ab-

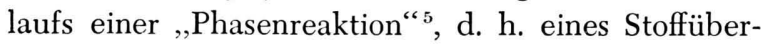
gangs zwischen den Phasen ohne Änderung von Druck, Temperatur und Konzentrationen. Bei dieser Úbergangsreaktion setzen sich entweder $\alpha_{1}$ Mole der Phase' mit $\alpha_{2}$ Molen der Phase" zu 1 Mol der Phase "" um, oder es findet der umgekehrte Vorgang statt. Fall II besagt geometrisch, daß die drei Punkte
$\mathrm{P}^{\prime}, \mathrm{P}^{\prime \prime}, \mathrm{P}^{\prime \prime \prime}$ auf der durch die Parameter $\beta_{1}$ und $\beta_{2}$ charakterisierten Geraden liegen und führt somit zu derselben Bedingung wie die Gln. I; diese sind bequemer zu interpretieren, weil aus ihnen direkt das Abstandsverhältnis der drei Punkte ablesbar ist, das unmittelbar zum Massenverhältnis der Phasen führt.

Der Sonderfall Ia wird durch das Zusammenfallen zweier Punkte veranschaulicht. Es werden also die Zusammensetzungen zweier Phasen gleich. Es handelt sich dann entweder um einen kritischen Entmischungspunkt oder um einen kritischen Punkt Flüssigkeit-Dampf oder um einen ternären azeotropen Punkt. Den letzten Fall, der die zufällige Lage eines azeotropen Punktes auf der Entmischungskurve bedeuten würde, wollen wir nicht in Betracht ziehen. Das Gegenstück zu Ia ist Sonderfall II a, der einem binären System entspricht: das Extremum liegt auf einer der Dreiecksseiten.

Es ergibt sich also folgender Satz: Ein Maximum oder Minimum des Dampfdruckes bei konstanter Temperatur bzw. des Siedepunktes bei konstantem Druck im Dreiphasenkoexistenzgebiet tritt dann und nur dann auf, wenn entweder die Konzentration einer Komponente Null ist (binäres System), oder die Darstellungspunkte der beiden Flüssigkeiten und des Dampfes auf einer Geraden liegen, oder ein kritischer Punkt vorliegt.

Demnach findet man bei jeder Entmischungskurve mindestens ein Maximum und ein Minimum, nämlich an den Enden des Entmischungsgebiets, das ja beiderseits entweder durch eine Dreiecksseite oder durch einen kritischen Punkt begrenzt wird. Innerhalb des heterogenen Gebiets können Extrema fehlen oder (evtl. mehrmals) auftreten.

Hiermit sind die oben gewonnenen Erkenntnisse analytisch präzisiert. In bezug auf die Temperaturabhängigkeit des Extremdruckes (bzw. Druckabhängigkeit der Extremtemperatur) sei auf die frühere allgemeine Abhandlung ${ }^{5}$ verwiesen.

Es liegt nun nahe, zu fragen, ob es möglich ist, auch im heterogenen Gebiet „Destillationslinien“ einzuführen. In den meisten neueren Darstellungen werden sowohl der Begriff als auch die besonderen Eigenschaften der Destillationslinien von homogenen Dreistoffgemischen auf heterogene Dreistoffgemisch ohne Begründung übertragen. Die erste kritische Diskussion der Destillationslinien im heterogenen Gebiet stellen die qualitativen Úberlegungen von Reinders und d e $\mathrm{M}$ in j e ${ }^{18}$ dar. Anknüpfend an frühere Aus-

${ }_{18}$ W. Reinders u. C. H. de Minjer, Recueil Trav. chim. Pays-Bas 59, 207 [1940]. 
führungen ${ }^{5}$, wollen wir kurz zu dieser Frage Stellung nehmen.

Wir betrachten den Vorgang der ,einfachen Destillation“, d. h. der kontinuierlichen Verdampfung ohne Auftreten eines Rücklaufes.

Es seien $z^{\prime \prime}$-Mole der ersten Flüssigkeit mit $z^{\prime \prime \prime}$ Molen der zweiten Flüssigkeit im Gleichgewicht. Die Molzahlen der Komponenten 1 und 2 in den einzelnen flüssigen Phasen betragen also: $z^{\prime \prime} x_{1}{ }^{\prime \prime}, z^{\prime \prime} x_{2}{ }^{\prime \prime}$; $z^{\prime \prime \prime} x_{1}^{\prime \prime \prime}, z^{\prime \prime \prime} x_{2}^{\prime \prime \prime}$.

Wir setzen ferner:

$$
\begin{aligned}
z^{\prime \prime}+z^{\prime \prime \prime} & =z, \\
\frac{z^{\prime \prime} x_{i}^{\prime \prime}+z^{\prime \prime \prime} x_{i}^{\prime \prime \prime}}{z} & =x_{i}^{*}
\end{aligned}
$$

und nennen $x_{i}{ }^{*}$ den mittleren oder Bruttomolenbruch des flüssigen Phasenkomplexes. Die Gesamtmolzahl der Komponente $i$ in diesem Komplex ist mithin $z x_{i}{ }^{*}$.

Es möge nun eine beliebig kleine Menge, $d z$-Mole, des Gleichgewichtsdampfes der Zusammensetzung $x_{1}{ }^{\prime}, x_{2}{ }^{\prime}$ gebildet werden. Wegen der Erhaltung der Masse ergibt sich dann:

Es folgt:

$$
\begin{aligned}
& d\left(z x_{1}{ }^{*}\right)=x_{1}{ }^{\prime} d z, \\
& d\left(z x_{2}{ }^{*}\right)=x_{2}{ }^{\prime} d z .
\end{aligned}
$$

$$
d x_{1}^{*} / d x_{2}{ }^{*}=\left(x_{1}{ }^{\prime}-x_{1}^{*}\right) /\left(x_{2}{ }^{\prime}-x_{2}{ }^{*}\right) .
$$

Gl. (18) ist der für homogene Dreistoffgemische geltenden Gl. B (9)

$$
d x_{1} / d x_{2}=\left(x_{1}{ }^{\prime}-x_{1}\right) /\left(x_{2}^{\prime}-x_{2}\right),
$$

in der sich die ungestrichenen Größen auf die Flüssigkeit und die gestrichenen auf den Dampf beziehen, vollkommen analog. Deshalb ist die Definition von „Destillationslinien“ auch im heterogenen Gebiet sinnvoll. Diese Linien geben die Änderung der Zusammensetzung des flüssigen Phasenkomplexes während der einfachen Destillation an. Ein PunktP $\left(x_{1}{ }^{*}, x_{2}{ }^{*}\right)$ stellt die Totalzusammensetzung eines Komplexes aus zwei koexistenten flüssigen Phasen dar. Er liegt daher im Darstellungsdreieck auf der Verbindungsgeraden der Punkte $\mathrm{P}^{\prime \prime}\left(x_{1}{ }^{\prime \prime}, x_{2}{ }^{\prime \prime}\right)$ und $\mathrm{P}^{\prime \prime \prime}\left(x_{1}{ }^{\prime \prime}, x_{2}{ }^{\prime \prime}\right)$, die den beiden Flüssigkeiten entsprechen, und teilt diese Strecke im umgekehrten Verhältnis der Massen (gerechnet in Molen) der beiden flüssigen Schichten. Der Punkt $\mathrm{P}^{\prime}\left(x_{1}{ }^{\prime}, x_{2}{ }^{\prime}\right)$, der die Dampfzusammensetzung angibt, liegt an einer bestimmten Stelle innerhalb oder außerhalb des Entmischungsgebietes, wie wir es oben besprochen haben. Wenn wir demnach Gl. (18) als die Differentialgleichung der Destillationslinien im heterogenen Gebiet betrachten, so folgt, daß die Tangenten an diese Linien in einem beliebigen Punkt $\mathrm{P}$ die Geraden sind, die $\mathrm{P}$ mit dem zugehörigen Dampfpunkt $\mathrm{P}^{\prime}$ verbinden. Nennen wir diese Geraden kurz die Geraden $\mathrm{PP}^{\prime}$, so ergibt sich der Satz: Die Destillationslinien eines ternären Systems mit zwei flüssigen Phasen sind die Enveloppen der Geraden $P P^{\prime}$.

An die Stelle der Vielzahl der „Dampflinien“ bei homogenen Gemischen tritt bei heterogenen Gemischen die einzige Dampfkurve, auf der alle Punkte $\mathrm{P}^{\prime}$ liegen.

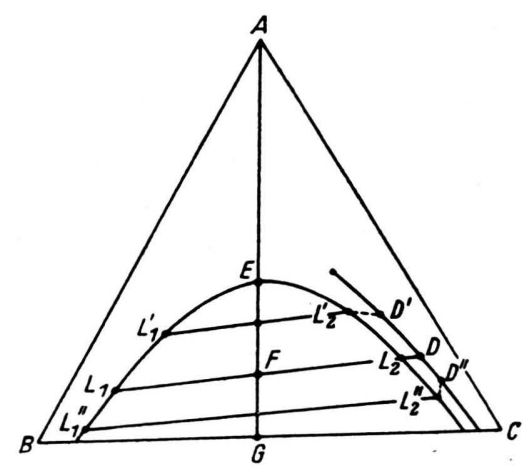

Abb. 6.

Auch bei der kontinuierlichen Verdampfung eines flüssigen Phasenkomplexes muß aus Gründen der Stabilität der Siedepunkt bei konstantem Druck monoton steigen. Daher verlaufen die Destillationslinien stets im Sinne steigender Temperatur, wenn - wie im folgenden immer - konstanter Druck, also isobare einfache Destillation, vorausgesetzt wird. Lediglich wenn es sich um ein System mit ausgezeichneter $\mathrm{Zu}$ sammensetzung (Maximum oder Minimum im heterogenen Gebiet) handelt, muß der Siedepunkt — solange zwei Schichten vorhanden sind - konstant bleiben, da in diesem Falle die kontinuierliche Verdampfung in einer Phasenreaktion besteht; die Destillationslinie entartet dann zu einer Geraden.

Tritt jedoch - wie bei der Rektifikation - ein Rücklauf auf, so entfallen die Voraussetzungen für die Anwendbarkeit der Stabilitätsbetrachtungen, da jetzt die Änderung der Zusammensetzung der in der Destillationsblase befindlichen Flüssigkeit nicht mehr allein durch Verschiebungen des Gleichgewichts bestimmt wird. Auf Grund unserer Überlegungen und Erfahrungen an homogenen Dreistoffgemischen ${ }^{3}$ werden wir daher erwarten, daß es Fälle gibt, bei denen 
während des Rektifikationsvorganges Sinken des Siedepunktes im Rückstande eintritt.

Betrachten wir als einfaches Beispiel die schematische Abb. 6. Aus den relativen Lagen der Dampfpunkte D, D', D" zu den Flüssigkeitspunkten $\mathrm{L}_{1}, \mathrm{~L}_{2}$, $\mathrm{L}_{1}{ }^{\prime}, \mathrm{L}_{2}{ }^{\prime}, \mathrm{L}_{1}{ }^{\prime \prime}, \mathrm{L}_{2}{ }^{\prime \prime}$ erkennt man, daß die Konnode $\mathrm{L}_{1} \mathrm{~L}_{2}$ einem Siedepunktsminimum entspricht. Aus der Lage des Punktes D schließen wir, daß dieser Minimumsiedepunkt nicht die niedrigste Temperatur des gesamten Systems sein kann (s. 3. Kap.). Den tiefsten Siedepunkt habe die reine Komponente A. Nehmen wir weiter an, das Gleichgewichtsdiagramm sei so beschaffen, daß bei idealer Rektifikation, d. h. mit unendlicher Bodenzahl, die reine Komponente A stets als Destillat erscheint, so ergibt sich, daß -

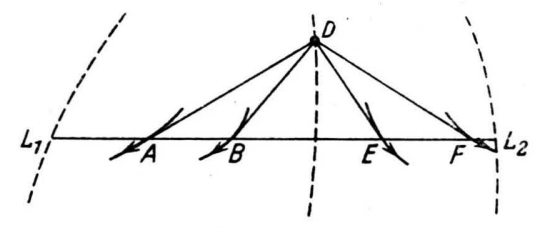

Abb. 7

bei Vernachlässigung des Kolonneninhaltes - der Rückstand in der Blase durch einen Punkt angegeben wird, der sich längs der Geraden AEFG in Richtung auf $\mathrm{G}$ zu bewegt. Zwischen $\mathrm{E}$ und $\mathrm{F}$ sinkt dann der Siedepunkt des Destillationsrückstandes. Dies ist - von weiteren Komplikationen des Gleichgewichtsdiagramms abgesehen - der Sachverhalt, den $\mathrm{Re}$ inder s und de M in je ${ }^{17}$ bei der Rektifikation des Systems Aceton-Chloroform-Wasser für Ausgangszusammensetzungen fanden, die durch Punkte der Geraden AG wenig oberhalb von $\mathrm{E}$ und zwischen $\mathrm{E}$ und $\mathrm{F}$ dargestellt werden, wenn reines Aceton (Punkt A) als Destillat erhalten wurde.

Abb. 7 gibt einen Ausschnitt eines Diagramms mit Teilen von vier Destillationslinien im heterogenen Gebiet. Die gestrichelten, durch $\mathrm{L}_{1}, \mathrm{~L}_{2}$ und D gehenden Kurven sind die beiden Teile der Entmischungskurve und die Dampfkurve, die gepfeilten Kurven die Destillationslinien. Die Flüssigkeiten $L_{1}$ und $L_{2}$ sind im Gleichgewicht mit dem Dampf D. Die Dampfkurve ist also hier als innerhalb des Entmischungsgebietes verlaufend angenommen. Gemäß der Definition der Destillationslinien berühren die Geraden DA, DB, DE und DF die vier Destillationslinien in den Punkten A, B, E und F. Dadurch kommt eine von Punkt zu Punkt variierende Richtung der Destillationslinien beim Fortschreiten längs der Konnode
$\mathrm{L}_{1} \mathrm{~L}_{2}$ zustande, obwohl alle durch Punkte der Strecke $\mathrm{L}_{1} \mathrm{~L}_{2}$ dargestellten Flüssigkeitskomplexe zu denselben Werten der Gleichgewichtskonzentrationen der koexistenten Phasen gehören. Diesen Umstand liest man auch aus Gl. (18) ab: Der Differentialquotient $d x_{1}{ }^{*} /$ $d x_{2}{ }^{*}$ ist bei gegebenen Werten von $x_{i}{ }^{\prime}, x_{i}{ }^{\prime \prime}$ und $x_{i}{ }^{\prime \prime}$ noch eine Funktion von $z^{\prime \prime} / z^{\prime \prime \prime}$, also dem Massenverhältnis der flüssigen Schichten.

Aus dem Gesagten können wir zwei wichtige Folgerungen ziehen. Einmal kommt ein ähnliches Bild des Destillationsfeldes wie bei den homogenen ternären Gemischen zustande, wobei ein auf einer Dreiecksseite zwischen den Flüssigkeitspunkten liegender Dampfpunkt (der stets zu einem tiefsten Siedepunkt des binären Systems gehört) einem binären azeotropen Punkt und ein Maximum oder Minimum im ternären heterogenen Gebiet einem ternären azeotropen Punkt entspricht. Andererseits erkennt man, daß die Destillationslinien mit der Dampfkurve allein das Gleichgewichtsdiagramm nicht ersetzen können, weil hieraus nicht die Zusammensetzungen der koexistenten flüssigen Phasen ablesbar sind. Daher haben die Destillationslinien heterogener Gemische nicht dieselbe fundamentale Bedeutung wie die Destillationslinien homogener Gemische, die zusammen mit den Dampflinien das System der Verdampfungsund Kondensationskurven mit den Konnoden ersetzen können.

\section{Si ed eflächen}

Errichtet man über jedem Punkt des Darstellungsdreiecks eine senkrechte Ordinate, die bei gegebenem $T$ dem Wert von $P$ bzw. bei gegebenem $P$ dem Wert von $T$ entspricht, so erhält man die Dampfdruckflächen bzw. Siedeflächen des ternären Systems. Wir beschränken uns auf die Betrachtung der Siedeflächen. Man kann unsere Überlegungen leicht sinngemäß auf die Dampfdruckflächen übertragen. Im homogenen Gebiet gibt es, wie früher ${ }^{2,3}$ ausführlich dargelegt, je eine Siedefläche für die Flüssigkeit und den Dampf; die letzte Fläche liegt stets oberhalb der ersten, außer in Eckpunkten des Darstellungsdreiecks (die den reinen Komponenten entsprechen) und in azeotropen Punkten (an denen Flüssigkeit und Dampf gleiche Zusammensetzung haben), wo beide Flächen zusammenfallen. Im heterogenen Gebiet muß die Siedefläche der Flüssigkeit eine besondere Gestalt haben; denn je zwei koexistente Flüssigkeiten haben denselben Wert von $T$ und liegen somit auf einer horizontalen Geraden, da alle in der Zusammensetzung zwischen den beiden Flüssigkeiten liegenden Ge- 
mische heterogen sind und sich in diese flüssigen Phasen spalten. Daraus folgt, daß die Siedefläche der Flüssigkeit im heterogenen Gebiet eine Regelfläche ist, die durch die Bewegung einer horizontalen $\mathrm{Ge}$ raden im Raume entsteht. Dies wird auch anschaulich, wenn man bedenkt, daß die Grenzkurve dieser Fläche über einer Dreiecksseite die horizontale Gerade im $T$ - $x$-Diagramm eines binären Systems mit Mischungslücke (vgl. IV, Abb. 2) ist. Aus dieser Veranschaulichung ersieht man ferner, daß die Siedefläche vom homogenen in das heterogene Gebiet mit „Knicken“ (Unstetigkeiten in den ersten Ableitungen) übergeht. Die zum Gebiet zweier Flüssigkeiten gehörenden Dampfpunkte liegen auf einer Raumkurve. Wie man wieder am besten durch eine gedachte räumliche Fortsetzung der für binäre Systeme mit Entmischung geltenden Siedekurven des Dampfes (Taukurven) erkennt, entsteht diese Raumkurve bei der Durchdringung der zwei die Siedefläche des Dampfes bildenden Teilflächen, die zu den beiden diesseits und jenseits der Regelfläche liegenden Siedeflächen der homogenen Flüssigkeiten gehören ${ }^{*}$. Den mit zwei Flüssigkeiten koexistenten Dampf findet man, indem man durch die Verbindungsgerade der beiden Flüssigkeitspunkte eine Horizontalebene legt; der Schnittpunkt mit der erwähnten Raumkurve ist dann der Dampfpunkt. Hieraus können wir sofort den Schluß ziehen, $\mathrm{da} ß$ bei einem Maximum oder Minimum des Siedepunkts im Dreiphasengebiet die räumliche Dampfkurve die Regelfläche bzw. die Verlängerung derselben berührt, da in einem solchen Falle die beiden Flüssigkeitspunkte und der Dampfpunkt auf einer Geraden liegen müssen.

Bei diesen Maxima oder Minima handelt es sich also nicht um Extrema in den Siedeflächen ${ }^{17}$, sondern um Maxima und Minima auf den räumlichen Dreiphasenkoexistenzkurven, d. h. sowohl auf der Raumkurve für den Dampf als auch auf den Kurven für die Flüssigkeiten, die als Grenzkurven zwischen dem homogenen und heterogenen Gebiet der Siedefläche bzw. als Durchdringungskurven der (nach beiden Seiten fortgesetzten) Regelfläche mit den Siedeflächen der homogenen Flüssigkeiten aufgefaßt werden können. Die fast überall benutzte Bezeichnung ,,azeotroper Punkt" für derartige Maxima und Minima ist daher als irreführend zu verwerfen, auch dann, wenn das Siedepunktsminimum der tiefste Siedepunkt des gesamten Systems ist. Die größte Zahl der

- Aus dem Vorhandensein zweier Teile der Dampfsiedefläche folgt auch, daß Kondensationskurven wie OHM in Abb. 2 einen Knickpunkt (H) aufweisen. in der Literatur angegebenen, ,ternären azeotropen Punkte" sind solche Minima im heterogenen Gebiet.

Es ist nun - besonders im Hinblick auf die Anwendungen in der Rektifiziertechnik - von Interesse, die Frage zu beantworten, ob und unter welchen Umständen ein Maximum oder Minimum im Entmischungsgebiet ein höchster oder tiefster Siedepunkt des gesamten Systems ist. Zu diesem Zwecke müssen wir die Siedepunkte der homogenen Gemische in der unmittelbaren Umgebung eines solchen Dreiphasensystems untersuchen.

Wir wenden einen von $\mathrm{S} c \mathrm{hr}$ ein e m a ke $\mathrm{r} \mathrm{s}^{19} \mathrm{ab}$ geleiteten Satz auf das vorliegende Problem an. Betrachten wir ein System $\Sigma$, bestehend aus drei Phasen, die sich, wie im obigen Falle, auf dem Wege einer Phasenreaktion reversibel umsetzen können, so entsteht durch reversible Wärmezufuhr ein bestimmtes Zweiphasensystem $\Sigma^{\prime}$ und durch Wärmeentzug ein anderes, das wir $\Sigma^{\prime \prime}$ nennen. Die Entropien des Dreiphasensystems, des ersten und des zweiten Zweiphasensystems bezeichnen wir mit $S, S^{\prime}$ und $S^{\prime \prime}$, die Freien Enthalpien bei der Temperatur $T$ mit $G, G^{\prime}$ und $G^{\prime \prime}$ und die Freien Enthalpien der Systeme $\Sigma^{\prime}$ und $\Sigma^{\prime \prime}$ bei der Temperatur $T+d T$ mit $\left(G^{\prime}\right)_{T+d T}$ und $\left(G^{\prime \prime}\right)_{T+d T}$. Die reversible Reaktion $\Sigma^{\prime \prime} \rightarrow \Sigma \rightarrow \Sigma^{\prime}$ verläuft bei Konstanz von Druck und Temperatur unter Wärmeaufnahme. Daher gilt:

$$
\begin{gathered}
G^{\prime \prime}=G=G^{\prime}, \\
S^{\prime}>S>S^{\prime \prime} .
\end{gathered}
$$

Von dem Augenblick an, in dem eine der Phasen dureh Zufuhr oder Entzug von Wärme aus dem Dreiphasensystem verschwunden ist, muß sich bei konstantem Druck die Gleichgewichtstemperatur ändern; sie steige von $T$ auf $T+d T$. Dann erhalten wir:

$$
\begin{aligned}
& \left(G^{\prime}\right)_{T+d T}=G^{\prime}+\frac{\partial G^{\prime}}{\partial T} d T^{\prime}=G^{\prime}-S^{\prime \prime} d T \\
& \left(G^{\prime \prime}\right)_{T+d T}=G^{\prime \prime}+\frac{\partial G^{\prime \prime}}{\partial T} d T=G^{\prime \prime}-S^{\prime \prime} d T .
\end{aligned}
$$

Mit (20) und (21) folgt aus (22) und (23):

$$
\left(G^{\prime \prime}\right)_{T+d T}>\left(G^{\prime}\right)_{T+d T^{\prime}}
$$

$\mathrm{Da}$ also das System $\Sigma^{\prime}$ bei gleichem Druck und gleicher Temperatur den kleineren Wert von $G$ hat, ist dieses System bei der Temperatur $T+d T$ stabil. Auf analoge Weise leitet man ab, daß bei der Temperatur $T-d T$ das System $\Sigma^{\prime \prime}$ stabil ist.

${ }^{19}$ H. W. B. R o o z e b o o $\mathrm{m}^{4}$, S. 296. 
Es ergibt sich also der Satz, daß durch Wärmezufuhr (Wärmeentzug) bei der Phasenreaktion sich dasjenige System bildet, das unter gleichem Druck bei höherer (niederer) Temperatur stabil ist.

Betrachten wir zuerst den Fall, daß die drei auf einer Geraden liegenden Punkte, die den beiden Flüssigkeiten $L_{1}$ und $L_{2}$ und dem Dampf $D$ entsprechen, eine Lage wie in Abb. 4 und 5 haben, so daß eine Phasenreaktion nach folgendem Schema eintreten kann:

$$
L_{1}+L_{2} \rightleftharpoons D
$$

worin die Zeichen $L_{1}, L_{2}$ und $D$ Phasen bestimmter Zusammensetzung und Menge bedeuten. Durch Wärmezufuhr muß sich Dampf bilden, so daß schließlich eins der beiden Zweiphasensysteme $L_{1}+D$ oder $L_{2}+D$ entsteht. Nach dem abgeleiteten Satz ist die zu diesen Systemen gehörige Gleichgewichtstemperatur höher als die Siedetemperatur des Dreiphasensystems. Andererseits kann durch Wärmeentzug nur das Zweiphasensystem $L_{1}+L_{2}$ entstehen, also ein System, das nicht mit Dampf im Gleichgewicht ist. Daher liegt der Siedepunkt des Systems $L_{1}+L_{2}+D$ bei gleichem Druck tiefer als die Siedepunkte der benachbarten homogenen Systeme, kann also, wenn es sich um ein Minimum im heterogenen Gebiet handelt, der tiefste Siedepunkt überhaupt sein. Wenn es sich jedoch um ein Maximum des Dreiphasengebiets handelt, so kann von einem höchsten Siedepunkt nicht die Rede sein.

Beispiele für den Fall eines Minimums, das zugleich dem tiefsten Siedepunkt des gesamten Systems entspricht, sind sehr zahlreich; am bekanntesten ist Äthanol-Benzol-Wasser. Das von Reinders und de Minjer ${ }^{17}$ untersuchte System Ameisensäure$m$-Xylol-Wasser ist ein Beispiel für das erwähnte Maximum im Dreiphasengebiet.

Ein zweiter Fall tritt ein, wenn der Punkt $D$ auf der Verlängerung der Geraden $L_{1} L_{2}$ liegt (s. Abb. 6). Auch hier kann es sich entweder um ein Maximum oder um ein Minimum im heterogenen Gebiet handeln. Anstelle von Gl. (25) lautet das Schema für die Phasenreaktion:

$$
L_{2} \rightleftharpoons L_{1}+D .
$$

Bei Wärmezufuhr bildet sich also aus einer Flüssigkeit die zweite Flüssigkeit und Dampf, so daß schließlich das System $L_{1}+D$ entsteht, das somit das bei höherer Temperatur beständige benachbarte Zweiphasensystem darstellt. Bei Wärmeentzug hingegen kann aus dem Dreiphasensystem - je nach der re- lativen Menge der Phasen - entweder das System $L_{2}+D$ oder $L_{1}+L_{2}$ hervorgehen. Es zeigt sich mithin, daß es hier sowohl bei höherer $\left(L_{1}+D\right)$ als auch bei tieferer Temperatur $\left(L_{2}+D\right)$ benachbarte Zweiphasensysteme mit Dampf gibt. Daher kann in diesem Falle ein Maximum im heterogenen Gebiet nicht dem höchsten, ein Minimum nicht dem tiefsten Siedepunkt des gesamten Systems entsprechen.

Ein interessantes Beispiel hierzu ist das von $\mathrm{R}$ e i $\mathrm{n}$ ders und de M in je r ${ }^{17}$ untersuchte System AcetonChloroform-Wasser, das im Entmischungsgebiet ein Maximum vom Typ (25) und ein Minimum vom Typ (26) aufweist; die Dampfkurve verläuft also hier teils innerhalb, teils außerhalb der Entmischungskurve.

Wir können die gewonnenen Erkenntnisse folgendermaßen zusammenfassen:

1. Ein Minimum des Siedepunkts im heterogenen Gebiet kann nur dann der tiefste Siedepunkt des gesamten ternären Systems sein, wenn die Dampfzusammensetzung zwischen den Zusammensetzungen der beiden flüssigen Schichten liegt.

2. Ein Maximum des Siedepunkts im heterogenen Gebiet kann nie der höchste Siedepunkt des gesamten ternären Systems sein.

Lecat ${ }^{20}$ sowie $\mathrm{Young}$ und $\mathrm{Prahl}^{21}$ bemerken bei ihren Zusammenstellungen azeotroper Daten, es seien noch keine ternären Systeme mit „Maximumsiedepunkt" bekannt. Auch in den neuen Tabellen von $\mathrm{H}$ or s le $\mathrm{y}^{22}$ ist ein solches System nicht zu finden. Nach den obigen Ausführungen müssen wir die Frage nach einem ,ternären Maximum“ in zwei Teilfragen zerlegen. a) Warum ist kein System mit höchstem Siedepunkt im heterogenen Gebiet bekannt? b) Warum fand man noch kein System mit Maximumsiedepunkt im homogenen Gebiet, d. h. mit echtem azeotropen Punkt, der einem Maximum in der Siedefläche entspricht?

Die Antwort auf Frage a) gibt der oben angeführte Satz: Einen solchen höchsten Siedepunkt gibt es prinzipiell nicht. Die Antwort auf Frage b) kann ebenfalls leicht gegeben werden: Ein ternäres Maximum im homogenen Gebiet ist prinzipiell möglich, wird aber fast ausschließlich nur dann vorkommen, wenn drei binäre Maxima vorhanden und die drei Komponenten in allen Verhältnissen mischbar sind. Da bei binären Systemen ein Siedepunktsmaximum viel seltener als

20 M. L e cat, La tension de vapeur des mélanges liquides, l'azéot:opisme, Brüssel 1918.

¿1 S. Young u. W. Prahl, Theorie und Praxis der Destillation, Berlin 1932.

22 L. H. H o r s ley, Analyt. Chemistry 19, 508-600 [1947]. 
ein Siedepunktsminimum ist, dürfte es sehr schwer fallen, eine Kombination dreier in allen Verhältnissen mischbarer Komponenten aufzufinden, bei der alle drei Stoffpaare ein binäres Maximum haben. Noch viel unwahrscheinlicher ist es daher, daß unter den bisher (meist nicht systematisch) auf einen ternären azeotropen Punkt untersuchten Dreistoffsystemen eines zu finden ist, das ein ternäres Maximum aufweist.

\section{Anhang}

Die Stabilitätsbedingungen

Bei der Ableitung der Gleichungen für den kritischen Punkt wurde von der Tatsache Gebrauch gemacht, daß für eine Phase, die aus $n$ Komponenten besteht, die Stabilitätsbedingungen bei gegebenen Werten von Druck und Temperatur auf zwei Weisen formuliert werden können. Erstens betrachtet man die totale Freie Enthalpie $g$ als Funktion der Molzahlen $m_{1}, m_{2} \ldots m_{n}$ und setzt $m_{n}=$ const; dann lautet die erste Formulierung der Bedingung für die Stabilität (innerhalb der Stabilitätsgrenzen):

$$
\begin{aligned}
& \Delta^{2} \boldsymbol{g}\left(m_{1}, m_{2} \ldots m_{n-1}\right)=\frac{1}{2}\left\{\frac{\partial^{2} g}{\partial m_{1}{ }^{2}}\left(\Delta m_{1}\right)^{2}\right. \\
& \left.+2 \frac{\partial^{2} g}{\partial m_{1} \partial m_{2}} \Delta m_{1} \Delta m_{2}+\ldots+\frac{\partial^{2} g}{\partial m_{n-1}{ }^{2}}\left(\Delta m_{n-1}\right)^{2}\right\}>0 .
\end{aligned}
$$

(27) ist gleichbedeutend mit der Bedingung, daß die Determinante

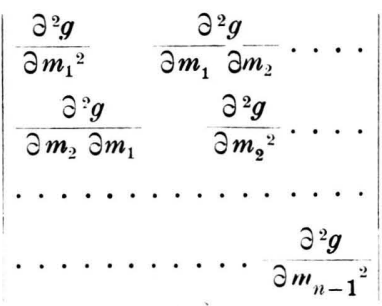

mit sämtlichen Hauptminoren positiv ist, woraus auch folgt, da $ß$ alle Diagonalglieder positiv sind. Zweitens setzt man: $m_{1}+m_{2} \cdots+m_{n}=$ const und führt die auf 1 Mol Mischung bezogene Freie Enthalpie (mittlere molare Freie Enthalpie)

$$
G=\frac{g}{m_{1}+m_{2}} \frac{g+m_{n}}{\cdots}
$$

und den Molenbruch

$$
x_{k}=\frac{m_{k}}{m_{1}+m_{2} \cdots+m_{n}}
$$

ein; dann lautet die zweite Formulierung der Bedingung für die Stabilität (innerhalb der Stabilitätsgrenzen):

$$
\Delta^{2} G\left(x_{1}, x_{2} \ldots x_{n-1}\right)>0,
$$

wenn wir $x_{1}, x_{2}, \ldots x_{n-1}$ als unabhängige Molenbrüche betrachten. (30) ist gleichbedeutend mit der Bedingung, daß die Determinante

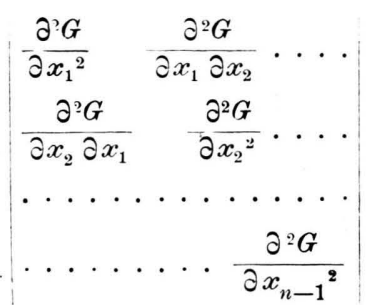

mit sämtlichen Hauptminoren positiv ist, woraus auch folgt, daß alle Diagonalglieder positiv sind.

Die Aquivalenz von (27) und (30) ist nicht so trivial, wie es zunächst scheint und offenbar in der Literatur ${ }^{23,24}$ angenommen wird. Es gilt nämlich [s. A, Gl. (10)]:

$$
\begin{gathered}
\left(\frac{\partial G}{\partial x_{i}}\right)_{P, T, x_{j}}=\mu_{i}-\mu_{n}, \\
(j=1,2 \ldots i-1, i+1 \ldots n-1),
\end{gathered}
$$

worin $\mu_{n}$ das chemische Potential derjenigen Komponente bedeutet, deren Molenbruch nicht als unabhängige Variable auftritt.

Mit der Beziehung

$$
\frac{\partial \mu_{i}}{\partial m_{k}}=\frac{\partial \mu_{i}}{\partial x_{k}} \frac{d x_{k}}{d m_{k}}=\frac{1-x_{k}}{\sum_{r=1}^{n} m_{r}} \frac{\partial \mu_{i}}{\partial x_{k}}
$$

und Gl. (6) folgt der Zusammenhang:

$$
\frac{\partial^{2} G}{\partial x_{i} \partial x_{k}}=\frac{\sum_{r=1}^{n} m_{r}}{1-x_{k}}\left(\frac{\partial^{2} g}{\partial m_{i} \partial m_{k}}-\frac{\partial^{2} g}{\partial m_{n} \partial m_{k}}\right) .
$$

Hieraus ergibt sich, daß - außer für $n=2-$ die Äquivalenz von (27) und (30) keineswegs selbstverständlich ist.

(27) ist implizit in den Schriften von Gibb s ${ }^{11}$ enthalten. Die für praktische Zwecke wichtigere Bedingung (30) ist für zwei Komponenten von va n d e r W a a l s ${ }^{25}$, für drei Komponenten auf geometrischem Wege von demselben Autor ${ }^{26}$ und $\mathrm{S} \mathrm{ch} \mathrm{re} \mathrm{in} \mathrm{e} \mathrm{make} \mathrm{r} \mathrm{s}{ }^{4}$, für beliebig viele Komponenten algebraisch vom Verf. ${ }^{2}$ hergeleitet worden; dabei wurde allerdings nur die Stabilität der Phase in bezug auf den Zerfall einer endlichen Menge der betrachteten Phase in zwei neue Phasen benachbarter Zusammensetzung untersucht, während die Stabilitätskriterien von $\mathrm{G}$ i b b s völlig allgemein sind. Eine befriedigende Lösung des Problems ist also erst gefunden, wenn (27) und (30) aus den ursprünglichen Gibbsschen Gleichungen abgeleitet werden können. Diese mathematischen Umformungen sollen uns hier beschäftigen, wobei die

23 A Commentary on the Scientific Writings of J. W. Gibbs I, S. 152, 171, New Haven 1936.

$: 4$ W. S chottky, H. Ulich u. C. Wagner, Thermodynamik, S. $447 \mathrm{ff}$., Berlin 1929.

${ }_{25}$ J. D. van der Wa als, Die Kontinuität des gasförmigen und flüssigen Zustandes, 2. Teil, Leipzig 1900.

${ }^{26} \mathrm{~J}$. D. van der Wa als, Arch. néerl. Sci. exact. natur. (2) 7, 343 [1902]. 
Stabilitätsbedingungen sogleich in ihrer allgemeinsten Form aufgestellt werden sollen.

Eine Phase heißt ,stabil in bezug auf kontinuierliche Änderungen“, wenn durch Bildung einer beliebigen Menge und Anzahl benachbarter Phasen innerhalb oder aus der ursprünglichen Phase die thermodynamischen Gleichgewichtsbedingungen verletzt würden. Unter „benachbarten Phasen “ versteht man solche, die sich in ihren Eigenschaften beliebig wenig von der betrachteten Phase unterscheiden. Eine Phase, die nur in bezug auf diskontinuierliche Änderungen instabil ist, heißt „metastabil“; eine solche, die in bezug auf kontinuierliche Änderungen instabil und daher überhaupt nicht existenzfähig ist, nennen wir „labil“. Im folgenden verstehen wir unter „Stabilität“ stets Stabilität in bezug auf kontinuierliche Änderungen; metastabile Phasen sind also durch die nachfolgenden Kriterien nicht von den übrigen stabilen Phasen zu unterscheiden.

Wir folgen zunächst den Ausführungen von Gibbs ${ }^{11}$. Betrachten wir den Ausdruck

$$
e-T s+P v-\mu_{1} m_{1} \ldots-!_{n} m_{n},
$$

worin $e$ die (totale) Energie, $s$ die (totale) Entropie, $v$ das (totale) Volumen, $m_{1} \ldots m_{n}$ die Massen (Molzahlen), $T$ die Temperatur der ursprünglichen Phase, $P$ den Druck der ursprünglichen Phase, $\mu_{1} \ldots \mu_{n}$ die chemischen Potentiale der ursprünglichen Phase bedeuten. Beziehen wir $e, s, v$, $m_{1} \ldots m_{n}$ auf die ursprüngliche Phase, so wird obiger Ausdruck identisch gleich Null. G i b b s ${ }^{11,23}$ hat gezeigt, daß eine Phase dann stabil ist, wenn dieser Ausdruck für jede benachbarte Phase positiv ist, wobei $e, s, v, m_{1} \ldots m_{n}$ sich jetzt auf die benachbarte Phase beziehen. Entsprechend ist die betrachtete Phase labil, wenn obiger Ausdruck für irgendeine benachbarte Phase negativ ist.

Bezeichnen wir die Größen, die sich auf die zu untersuchende Phase beziehen, durch einfach gestrichene Symbole und die Größen, die sich auf die andere Phase beziehen, durch doppelt gestrichene Symbole, so kann die Stabilitätsbedingung geschrieben werden:

$$
e^{\prime \prime}-T^{\prime} s^{\prime \prime}+P^{\prime} v^{\prime \prime}-\mu_{1}^{\prime} m_{1}^{\prime \prime} \ldots-\|_{n}{ }^{\prime} m_{n}{ }^{\prime \prime}>0 .
$$

Subtrahieren wir die Gleichung

$$
e^{\prime}-T^{\prime} s^{\prime}+P^{\prime} v^{\prime}-\prime_{1}^{\prime} m_{1}^{\prime} \ldots-\mu_{n}^{\prime} m_{n}^{\prime}=0
$$

von (33), so erhalten wir:

$e^{\prime \prime}-e^{\prime}>T^{\prime}\left(s^{\prime \prime}-s^{\prime}\right)-P^{\prime}\left(v^{\prime \prime}-r^{\prime}\right)+\prime_{1}^{\prime}{ }^{\prime}\left(m_{1}{ }^{\prime \prime}-m_{1}^{\prime}\right) \ldots+!_{n}^{\prime}$. $\left(m_{n}{ }^{\prime \prime}-m_{n}{ }^{\prime}\right)$

oder abgekürzt :

$$
\Delta e>T \Delta s-P \Delta v+!_{1} \Delta m_{1} \ldots+!_{n} \Delta m_{n} .
$$

Hierin bedeutet das Zeichen $\Delta$, daß die Bedingung, obwohl für beliebig kleine Änderungen gültig; nicht in der bei Differentialgleichungen üblichen Art mit Vernachlässigung infinitesimaler Größen höherer Ordnung, sondern streng zu interpretieren ist. (35) muß innerhalb der Stabilitätsgrenzen für jedes Páar benachbarter Phasen gelten.

Die Energie $e$ ist die charakteristische Funktion für die unabhängigen Variablen $s, v, m_{1} \ldots m_{n}$ :

$$
d e=T d s-P d v+\mu_{1} d m_{1} \ldots+!_{n} d m_{n} .
$$

Auf der rechten Seite von (35) steht also die Änderung erster Ordnung von $e$. Beschränken wir unsere Diskussion auf Änderungen der Funktionen bis zur zweiten Ordnung, so folgt aus (35):

$$
A^{2} e\left(s \cdot v \cdot m_{1} \ldots m_{n}\right)>0 .
$$

worin $\Delta^{2} e$ die Änderung zweiter Ordnung von $e$ bedeutet.

Schottky, Ulich und Wagner legen ihren Betrachtungen über Stabilität die Bedingung (36) zugrunde, ohne für diese die exakte Begründung zu geben, die man bei $\mathrm{Gib}$ b findet, und deren letzten Teil wir hier wiedergegeben haben.

Es ist zu bẹachten, daß alle Variationen, bei denen sich nur die Menge der Phase ändert, auszuschließen sind. Wir können dies dadurch berücksichtigen, daß wir entweder wie Gibbs - das Volumen oder die Masse (Molzahl) irgendeiner Komponente oder die Summe aller Massen als konstant annehmen.

Setzen wir zunächst $m_{n}=$ const, so erhalten wir als Stabilitätsbedingung:

$$
\left[\Delta^{2} e\left(s, v, m_{1} \ldots m_{n-1}\right)\right]_{m_{u}}>0 .
$$

Diese Ungleichung kann man natürlich auch in Determinantenform schreiben.

Setzen wir sodann $m_{1}+m_{2} \ldots+m_{n}=$ const, so ergibt sich aus (35):

$$
\begin{gathered}
\Delta E>T \Delta S-P \Delta V+\mu_{1} \Delta x_{1} \ldots \\
\quad \ldots+\mu_{n-1} \Delta x_{n-1}+\mu_{n} \Delta x_{n} .
\end{gathered}
$$

worin $E, S, V$ die Energie, die Entropie und das Volumen je Mol Mischung sind. Mit Gl. (29) finden wir für den abhängigen Molenbruch $x_{n}$ :

$$
\Delta x_{n}=-\left(\Delta x_{1}+\Delta x_{2} \ldots+\Delta x_{n-1}\right) .
$$

Somit erhalten wir:

$$
\begin{aligned}
\Delta E>T \Delta S-P \Delta V+ & \left(!_{1}-\mu_{n}\right) \Delta x_{1} \ldots \\
& \ldots+\left(!_{n-1}-!_{n}\right) \Delta x_{n-1} .
\end{aligned}
$$

Aus $d E=T d S-P d V+\sum_{i=1}^{n} \prime_{i} d x_{i}$

$$
=T d S-P d V+\sum_{i=1}^{n-1} \frac{\partial E}{\partial x_{i}} d x_{i}
$$

folgt: $\left(\frac{\partial E}{\partial S}\right)_{V, x_{k}}=T \cdot\left(\frac{\partial E}{\partial V}\right)_{s, x_{k}}=-P$.

$$
\left(\frac{\partial E}{\partial x_{i}}\right)_{S, V, x_{j}(j \neq i)}=\mu_{i}-\mu_{n} .
$$

Es ergibt sich also, daß die rechte Seite von (39) die Änderung erster Ordnung der Funktion $E\left(S, V, x_{1} \ldots x_{n}, 1\right)$ darstellt. Es folgt die Stabilitätsbedingung:

$$
\Delta E\left(S V . x_{1} \ldots x_{n-1}\right)>0 .
$$

Auch diese Ungleichung kann leicht in Determinantenform geschrieben werden. 
Die Kriterien (37) und (40) sind einander gleichwertig.

Die Stabilitätsbedingungen seien jetzt durch die übrigen charakteristischen Funktionen ausgedrückt.

Für die Enthalpie

$$
h=e+P v=T s+\mu_{1} m_{1} \ldots+\mu_{n} m_{n}
$$

leiten wir aus (33) ab:

$$
\begin{gathered}
{[\Delta h-v \Delta P]_{s, m_{i}}<0 \quad(i=1,2 \ldots n),} \\
{\left[\Delta h-T \Delta s-\mu_{1} \Delta m_{1} \ldots-\mu_{n} \Delta m_{n}\right]_{P}>0 .}
\end{gathered}
$$

Mit der Beziehung

$$
d h=T d s+v d P+\mu_{1} d m_{1} \ldots+\mu_{n} d m_{n}
$$

ergibt sich:

$$
\begin{gathered}
\left.\Delta^{2} h(P)\right]_{s, m_{i}}<0 . \\
{\left[\Delta^{2} \dot{h}\left(s, m_{1} \ldots m_{n-1}\right)\right]_{P, m_{n}}>0 .}
\end{gathered}
$$

Bezeichnen wir die auf 1 Mol Mischung bezogenen Funktionen (mittleren molaren Größen) wieder mit groBen Buchstaben, so folgt durch eine dem obigen analoge Schlußweise, daß nachfolgende Bedingungen mit (41) und (42) äquivalent sind:

$$
\begin{gathered}
{\left[\Delta^{2} H(P)\right]_{S, x_{i}}<0 \quad(i=1,2 \ldots n-1),} \\
\left.\Delta^{2} H\left(S, x_{1} \ldots x_{n-1}\right)\right]_{P}>0 .
\end{gathered}
$$

Für die Freie Energie

$$
f=e-T s=-P v+\mu_{1} m_{1} \ldots+\iota_{n} m_{n}
$$

leitet man aus (33) ab:

$$
\begin{gathered}
{[\Delta f+s \Delta T]_{\ell, m_{i}}<0 .} \\
{\left[\Delta f+P \Delta \imath-\mu_{1} \Delta m_{1} \ldots-\mu_{n} \Delta m_{n}\right]_{T}>0 .}
\end{gathered}
$$

Diese beiden Ungleichungen finden sich bereits bei Gibbs.

Mit der Beziehung

$$
d f=-s d T-P d r+\mu_{1} d m_{1} \ldots+\mu_{n} d m_{n}
$$

ergibt sich:

$$
\begin{gathered}
{\left[\Delta^{2} f(T)\right]_{v, m_{i}}<0,} \\
{\left[\Delta^{2} f\left(v, m_{1} \ldots m_{n-1}\right)\right]_{T, m_{n}}>0 .}
\end{gathered}
$$

Es läßt sich weiter auf analogem Wege wie für $E$ und $H$ zeigen, daß nachfolgende Bedingungen mit (45) und (46) äquivalent sind ( $F=$ mittlere molare Freie Energie):

$$
\begin{gathered}
{\left[\Delta^{2} F^{\prime}(T)\right]_{V, x_{i}}<0,} \\
\left.A^{2} F\left(V, x_{1} \ldots x_{n-1}\right)\right]_{T}>0 .
\end{gathered}
$$

Aus (48) folgen für $n=2$ die von va n der W a ls und $\mathrm{K}$ or $\mathrm{t}$ e w e g geometrisch ausführlich diskutierten Bedingungen:

$$
\left|\begin{array}{cc}
\frac{\partial^{2} \boldsymbol{F}}{\partial} \boldsymbol{V}^{2} & \frac{\partial^{2} \boldsymbol{F}}{\partial \boldsymbol{V} \partial \boldsymbol{x}} \\
\frac{\partial^{2} \boldsymbol{F}}{\partial \boldsymbol{V}} \overline{\partial \boldsymbol{x}} & \frac{\partial^{2} \boldsymbol{F}}{\partial \boldsymbol{x}^{2}}
\end{array}\right|>0, \quad \frac{\partial^{2} \boldsymbol{F}}{\partial \boldsymbol{V}^{2}}>0 . \quad \frac{\partial^{2} \boldsymbol{F}}{\partial \boldsymbol{x}^{2}}>0 .
$$

Für die Freie Enthalpie

$$
g=e+P v-T s=\mu_{1} m_{1} \ldots+\mu_{n} m_{n}
$$

leiten wir aus (33) ab:

$$
\begin{gathered}
{[\Delta g+s \Delta T-v \Delta P]_{m_{i}}<0,} \\
{\left[\Delta g-\mu_{1} \Delta m_{1} \ldots-\mu_{n} \Delta m_{n}\right]_{T, P}>0 .}
\end{gathered}
$$

Diese beiden Ungleichungen finden sich bereits bei Gib bs.

Mit der Beziehung

$$
d g=-s d T+v d P+\mu_{1} d m_{1} \ldots+\mu_{n} d m_{n}
$$

ergibt sich:

$$
\begin{gathered}
{[\Delta g(T, P)]_{m_{i}}<0,} \\
{\left[\Delta^{2} g\left(m_{1}, m_{2} \ldots m_{n-1}\right)\right]_{T, P, m_{n}}>0 .}
\end{gathered}
$$

(50) bedeutet, daß

$$
\left|\begin{array}{cc}
\frac{\partial^{2} g}{\partial T^{2}} & \frac{\partial^{2} g}{\partial T \partial P} \\
\frac{\partial^{2} g}{\partial T \partial P} & \frac{\partial^{2} g}{\partial P^{2}}
\end{array}\right|>0, \quad \frac{\partial^{2} g}{\partial T^{2}}<0 . \quad \frac{\partial^{2} g}{\partial P^{2}}<0 .
$$

Die beiden letzten Ungleichungen führen zu den bekannten Bedingungen:

$$
\frac{\partial s}{\partial T}>0, \frac{\partial v}{\partial P}<0 .
$$

(51) ist identisch mit (27). Damit sind wir zu unserem Ausgangspunkt zurückgekehrt. Wir haben (27) aus den ursprünglichen $\mathrm{G}$ i b b s schen Gleichungen abgeleitet. Der Beweis der Äquivalenz von (27) und (30) sei, obwohl im analogen Falle für die Funktion $e$ schon erledigt, an dieser Stelle ausführlich gebracht.

Aus

$$
\Delta g>\mu_{1} \Delta m_{1} \ldots+\mu_{n} \Delta m_{n}
$$

folgt für $m_{1}+m_{2} \ldots+m_{n}=$ const :

$$
\Delta G>\mu_{1} \Delta x_{1} \ldots+\mu_{n-1} \Delta x_{n-1}+\mu_{n} \Delta x_{n} .
$$

Mit (38) ergibt sich:

$$
\Delta G>\left(\mu_{1}-\mu_{n}\right) \Delta x_{1} \ldots+\left(\mu_{n-1}-\mu_{n}\right) \Delta x_{n-1} .
$$

Aus Gl. (31) sehen wir, daß die rechte Seite von (52) die Änderung erster Ordnung der Funktion $G$ ist.

Daher erhalten wir schließlich:

$$
\left[\Delta^{2} G\left(x_{1}, x_{2} \ldots x_{n-1}\right)\right]_{T, P}>0 .
$$

(53) ist identisch mit (30); damit ist die Äquvalenz von (27) und (30) bewiesen. - Ferner läßt sich sofort zeigen, $\mathrm{da}$ anstelle von (50) gesetzt werden darf:

$$
\left[\Delta^{2} G(T, P)\right]_{x_{i}}<0 .
$$

Indem wir (41) und (42), (45) und (46) sowie (50) und (51) jeweils zu einer Bedingung zusammenziehen und (37) 


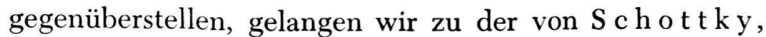
Ulich und Wagner ${ }^{24}$ aufgestellten allgemeinsten Stabilitätsbedingung für eine beliebige charakteristische Funktion $\varphi$ mit den zugehörigen Extensitätsparametern $\eta_{1}, \eta_{2} \ldots \eta_{k}$ und den Intensitätsparametern $y_{1}, y_{2} \ldots$ $y_{n+2-k}:$

$$
\begin{aligned}
{\left[\Delta^{2} \varphi\left(\eta_{1} \cdot \eta_{2} \ldots \eta_{k-1}\right)\right]_{y_{1}, y_{2} \ldots \eta_{k}} } & \\
& -\left[\Delta^{2} \varphi\left(y_{1}, y_{2} \ldots\right)\right]_{\eta_{1}, \eta_{2} \ldots \eta_{k}}>0 .
\end{aligned}
$$

Führen wir endlich die mittlere molare, d. h. die auf $1 \mathrm{Mol}$ Mischung bezogene charakteristische Funktion $\Phi$ ein und bezeichnen die ,spezifischen“ Extensitätsparameter (z. B. mittleres Molvolumen, Molenbrüche) mit $\bar{\eta}_{i}$, so erhalten wir aus (40), (43), (44), (47), (48), (53) und (54) die mit
(55) gleichwertige Stabilitätsbedingung:

$$
\begin{aligned}
{\left[\Delta^{2} \Phi\left(\bar{\eta}_{1}, \bar{\eta}_{2} \ldots \bar{\eta}_{k-1}\right)\right]_{y_{1}, y_{2}} \ldots } & \\
& -\left[\Delta^{2} \Phi\left(y_{1}, y_{2} \ldots\right)\right]_{\eta_{1}, \eta_{2} \ldots \eta_{k-1}}>0 .
\end{aligned}
$$

Ungleichung (56), die für die Anwendung wichtiger als (55) ist, haben wir hiermit streng abgeleitet. Alle bislang bei speziellen thermodynamischen Diskussionen benutzten Stabilitätsbedingungen, z. B. (49) und (53), sind Spezialfälle von (56). Eine exakte Ableitung dieser Bedingungen ist in der Literatur nicht zu finden.

Hrn. Prof. W. J o s t danke ich für das Interesse an der Arbeit und dafür, daß er die Durchführung dieser Untersuchungen in seinem Institut ermöglicht hat.

\title{
Ein Beitrag zum Eiszeitproblem II
}

\author{
Von KuRt Himpel \\ Aus dem Institut für angewandte Physik der Universität Frankfurt a. M. \\ (Z. Naturforschg. 5 a, 124-126 [1950]; eingegangen am 20. September 1948)
}

\begin{abstract}
An Hand neuerer paläoklimatologischer Forschungsergebnisse wird nachgewiesen, daß sowohl die Polwanderungstheorie als auch die astronomische Theorie von Milankovitch in bezug auf die Verhältnisse während des Känozoikums und namentlich während des Alluviums versagen. Es wird weiterhin ausgeführt, daß die Nebelveränderlichkeit nicht etwa durch rein optische Bedeckungseffekte, sondern durch die Bildung einer dichten, den Stern umgebenden Hülle, welche den Energietransport in der Sternatmosphäre stört, hervorgerufen wird. Die kleineren Schwankungen innerhalb der Eiszeiten (Interstadialzeiten) und die kurzdauernden Schwankungen des Alluvialklimas können durch die faserige (Filament-) Struktur der Dunkelwolken erklärt werden.
\end{abstract}

$I_{\mathrm{d}=0}^{n}$ $n$ den letzten $1 \frac{1}{2}$ Jahren sind, in den verschiedensten Zeitschriften verstreut, eine Reihe grundlegender Arbeiten auf dem Gebiete der Paläoklimatologie erschienen, daß es lohnend erscheint, vor einem breiteren Forum auf sie hinzuweisen und ihre neue Tendenz herauszustellen.

M. S chw a r z b a ch ${ }^{\mathbf{1}}$ kommt in seiner zusammenfassenden Untersuchung über die Klimazeugen des Alttertiär ${ }^{2}$ zu dem bemerkenswerten Ergebnis, daß die damalige Lage von Polen und Kontinenten der heutigen ähnlich gewesen sein dürfte. Bisher herrschte in Geologenkreisen immer noch die von Max Se mpe $r$ in seinen klassisch gewordenen Arbeiten vertretene Ansicht vor, daß wir im Alttertiär mit einer größeren Polverschiebung zu rechnen hätten; aber das seit über einer Generation (1912) mächtig angewachsene Beobachtungsmaterial hat dem nicht recht gegeben. Schwarzbachs Hauptresultat ist die Annahme einer bedeutend wärmeren, eisfreien Erde im Alttertiär. Seine Ansicht, dieser Zustand könne mit

1 M. S c h w a r z b a c h, Naturwiss. 33, 355 [1947].

$\because$ Das Alttertiär (Paläozän und Eozän) ist so ziemlich die wärmste Zeit der ganzen Erdgeschichte und deswegen hier besonders wichtig. terrestrischen Faktoren allein nicht erklärt werden, verdient unsere besondere Beachtung.

Eine besonders wertvolle Studie hat Schwar z $\mathrm{b} a \mathrm{ch}$ zwei neuen Büchern von F. E. Z e u n e $\mathrm{r}^{3}$ gewidmet, von denen uns besonders das Buch über das Eiszeitklima angeht. Schwarzbach kommt zu dem Ergebnis, daß trotz des enormen Forschungsaufwandes die Milankovitchsche Theorie von der Geologie immer noch nicht als bewiesen angesehen werden kann.

Hieran läßt sich eine gleichfalls bedeutsame Studie von $\mathrm{Fi} \mathrm{rbas}{ }^{4}$ über das Klima des Postglazial, zu dessen besten Kennern dieser Forscher zählt, anschließen. Nach ihm sind die Differenzen zwischen der Beobachtung und der Milankovitchschen Theorie doch viel größer als man bisher annahm, so daß günstigstenfalls „,noch eine halbe Eiszeit“ zu erklären übrig bleibt.

Auch Parets ausgezeichnetes Werk ${ }^{5}$ wird der Altklimatologe gerne studieren. Sein für uns besonders wichtiges Ergebnis ist, daß der relativ gleich-

${ }^{3}$ M. S c h w a r z b a c h, Geol. Rdsch. 1948, Heft 2/3.

${ }_{4}^{4}$ H. F i r b a s, Naturwiss. 34, 104 [1947].

¿ P a r e t, Das neue Bild der Vorgeschichte, Stuttgart 1946. 